\title{
Circular RNAs: Crucial regulators in the human body (Review)
}

\author{
YUANYONG WANG $^{1}$, TONG LU ${ }^{1}$, QIAN WANG ${ }^{2}$, JIA LIU $^{3}$ and WENJIE JIAO ${ }^{1}$ \\ ${ }^{1}$ Department of Thoracic Surgery, Affiliated Hospital of Qingdao University, Qingdao 266003; ${ }^{2}$ College of Nursing, \\ Weifang Medical University, Weifang 261053; ${ }^{3}$ School of Pharmacy, Qingdao University, Qingdao 266003, P.R. China
}

Received April 29, 2018; Accepted September 7, 2018

DOI: $10.3892 /$ or.2018.6733

\begin{abstract}
Circular RNAs (circRNAs) belong to a new type of endogenous non-coding RNAs (ncRNAs) that are derived from exons and/or introns, and are widely distributed in mammals. The majority of circRNAs have a specific expression profile in cells or tissues, as well as during different stages of development. CircRNAs were originally thought to be the products of mis-splicing. However, with the assistance of bioinformatics tools and the rapid development of high-throughput sequencing, an increasing body of evidence has suggested that circRNAs bind micro(mi)RNAs, and have a role as miRNA sponges, thereby regulating target mRNA splicing and transcription. Human diseases are closely associated with circRNAs, especially in cancer as their expression is typically altered during the progression of cancer; this may provide a novel type of biomarker for cancer diagnosis and prognosis. CircRNAs are becoming a key area of interest within the field of cancer research. In the present review, we summarize the known molecular mechanisms and biological
\end{abstract}

Correspondence to: Professor Wenjie Jiao, Department of Thoracic Surgery, Affiliated Hospital of Qingdao University, Jiangsu Road, Qingdao 266003, P.R. China

E-mail: jiaowj@qduhospital.cn

Abbreviations: circRNAs, circular RNAs; miRNAs, microRNAs; lncRNAs, long non-coding RNAs; ncRNAs, non-coding RNAs; RNA-Seq, RNA sequencing technology; RBP, RNA-binding protein; pre-mRNA, precursor messenger RNA; hnRNA, heterogeneous nuclear RNA; ecircRNAs, exonic circRNAs; ciRNAs, circular intronic RNAs; EIciRNAs, exon-intron circRNA; MREs, miRNA response elements; ceRNAs, competitive endogenous RNAs; ORFs, open reading frames; EV, extracellular vesicle; NSCLC, non-small cell lung cancer; CRC, colorectal cancer; ESCC, esophageal squamous cell carcinoma; DCIS, ductal carcinoma in situ; HCC, hepatocellular carcinoma; PDAC, pancreatic ductal adenocarcinoma; cSCC, cutaneous squamous cell carcinoma; LSCC, laryngeal squamous cell cancer; APL, acute promyelocytic leukemia; PTC, papillary thyroid cancer; IHD, ischaemic heart disease

Key words: circRNA, cancer, miRNA sponges, transcription, biomarkers, database origin of circRNAs, as well as their functions, especially those related to human tumors.

\section{Contents \\ 1. Introduction \\ 2. Biogenesis of circRNAs \\ 3. Characteristics of circRNAs \\ 4. Biofunctions of circRNAs \\ 5. Metabolism of circRNAs \\ 6. Public databases on circRNAs \\ 7. CircRNAs and human disease \\ 8. CircRNAs and cancer \\ 9. Conclusions \\ 10. Perspectives}

\section{Introduction}

To date, short microRNAs (miRNAs/miRs), long non-coding RNAs (lncRNAs) and circular RNAs (circRNAs), which together comprise non-coding RNAs (ncRNAs), have been validated $(1,2)$. They account for $~ 95 \%$ of the total RNA in eukaryotic transcription $(3,4)$. CircRNAs are produced from the back-splicing of exons and/or introns, forming exonic or intronic circRNAs, respectively (3). As a novel type of endogenous ncRNAs, circRNAs were first observed more than 40 years ago by Sanger et al (4-6). Originally, they were thought to be errors of splicing without any specific function and to be fairly rare (7-9), as circRNAs have only been identified in a few mammalian genes, such as ETS proto-oncogene 1 transcription factor (10) and sex determining region Y (SRY) (11). Subsequently, an increasing number of circRNAs have been discovered, and their roles in cancer have been determined through advances in high-throughput RNA sequencing technology (RNA-Seq) and bioinformatic methods $(11,12)$. There is also an increasing interest in their biofunction as miRNA sponges $(7,13-15)$, in regulating gene expression and transcription $(7,16)$, and also as RNA-binding protein (RBP) sponges (17-19). Cell proliferation, migration, invasion and metastasis are also related to the function of circRNAs; therefore, they may potentially be neoteric biomarkers and remedial targets for cancer therapy (20-23). In recent years, some previous studies have reported that circRNAs are stable, abundant and conserved, presenting tissue- and cell-specific expression patterns (24-26). 
In the present review, we briefly delineate the biogenesis, characteristics and biofunctions of circRNAs. We then utilize databases such as CircNet to evaluate known circRNAs, and how they are involved in specific processes and human diseases such as cancer. Furthermore, we discuss how circRNAs combine with miRNA sponges, regulating gene transcription and expression, and with RBP sponges in different cancers, confirming the clinical value of circRNAs in tumor diagnosis, treatment and prognosis.

\section{Biogenesis of circRNAs}

On the basis of various biogenetic patterns, circRNAs can be divided into the following three categories: exonic circRNAs (EcircRNAs) (7), circular intronic RNAs (ciRNAs) (27) and exon-intron circRNAs (EIciRNAs) (16) (Fig. 1).

EcircRNAs. The most plentiful circRNAs are EcircRNAs. The majority are derived from the coding gene of pre-mRNA, but do not involve coding proteins $(7,28,29)$. Commonly, there is sequential splicing in eukaryotic cells resulting in the removal of non-coding introns by alternative splicing of pre-mRNAs after transcription; this generates connected exons containing protein-coding genes which form translatable directly linear RNAs that produce the relevant protein (30). Nevertheless, exon sequences of genes are linked end-to-end, namely the EcircRNAs shaped by back-splicing. According to published reviews, there are generally two models of back-splicing (7): Lariat-driven circularization and intron-pairing-driven circularization. The former suggests that during the transcription of pre-mRNA, the pre-mRNA is partially folded and then segmental splicing of the RNA [i.e. the splice donor of one exon is covalently connected with the different exon(s), shortening the distance between the original non-adjacent exons] gives rise to exon skipping and the shape of a circRNA model. Potentially, each of the exons can be skipped by transcription. Afterwards, the EcircRNA is produced through intra-lariat splicing (31).

The intron-pairing-driven circularization pattern has also been well established. In this pattern, exons involved in circularization are linked to introns containing reciprocal complementary sequences that produce spatially close and end-to-end splice exons to generate circRNAs. However, it is worth noting that not all circRNAs are produced by complementary sequences on either side of an exon. Wang and Wang (32) constructed a model of a minigene in vitro and concluded that the reciprocal complementation between upstream or downstream exon-flanking introns was not necessary for the production of circRNAs. In addition, in 2014, a negative correlation between linear mRNA splicing and back-splicing of circRNAs was identified by Ashwal-Fluss et al (33).

There is a common feature in both of these models described. Intron-pairing introns can trigger exon circularization (27). In addition, the human genome contains a mass of complementary sequences whose pairing can produce diverse circRNAs by alternative circularization (34).

Circular intronic RNAs (ciRNAs). Intron regions account for at least $20 \%$ of the human genome, and the majority form a lasso-like structure (28). However, several introns containing key nucleotide sequences via the formation of intron-derived ciRNAs, cannot debranch after splicing and replacing $(34,35)$. It has been well established that the formation of ciRNAs relies on conserved sequences at both ends of the intron. These sequences promote debranching and produce a circRNA (27). Additional protein factors that have an impact on the formation of ciRNAs have not been defined. The formation of ciRNAs is quite different from that of EcircRNAs. CiRNAs are comprised of 2'-5' phospholipid-linked nucleotides, and EcircRNAs are 3'-5'phospholipid-linked nucleotides (36). CiRNAs are located in the nuclei and are capable of binding and affecting the expression of the original genes (37).

EIcircRNAs. Within the back-splicing process that forms EcircRNAs, circRNAs containing unspliced introns are present and are stable (16). These introns possibly act as mediators of the splicing process, or produce specific circRNAs. By means of RNA sequencing and bioinformatics analysis, Li et al (38) discovered $>100$ circRNAs that interact with polymerase (Pol) II by presetting a RNA Pol II CLIP sample in human cells (immobilization of Pol II and RNA after immunoprecipitation of Pol II antibody). Further studies have reported that these types of circRNAs can also be formed via exon back-splicing; however, these circRNAs simultaneously contain exons and introns that are abundantly localized in the nuclei (39-41). Therefore, these distinct circRNAs were called retained-intron circRNAs.

\section{Characteristics of circRNAs}

According to the published literature, there are several important traits of circRNAs. i) Plentifulness: Salzman et al (29) first suggested that circRNAs are distributed more widely than linear RNAs in mammals. ii) Stability: As circRNAs are covalently closed loops, and thus protected from RNase R, it has also been proposed that they are more stable than linear mRNAs (29). iii) Conservation: circRNAs are highly conserved across different species. A number of circRNAs have been detected in humans, mice, fruit flies and even plants $(7,42)$. iv) Situation: circRNAs are primarily composed of EcircRNAs and are predominantly located in the cytoplasm and possibly in miRNA response elements (MREs) $(13,43)$. Intronic circRNAs including ciRNAs and EIciRNAs, are both mainly located in the nuclei, and can therefore regulate gene transcription or post-transcription (7,27). v) Expression: circRNAs display tissue and/or development-specific expression patterns. For example, circRNAs can be easily detected in mammals, especially during synaptic and neuronal differentiation, as their expression levels are markedly increased (44). vi) Functionality: A few circRNAs contain miRNA binding sites which can combine with miRNA in order to weaken the endogenous miRNA-mediated mRNA binding activities via competitive binding (7).

\section{Biofunctions of circRNAs}

CircRNAs can function as miRNA sponges, gene transcription and expression regulators, RBP sponges, and can also regulate gene expression levels to affect protein/peptide translators at either the transcriptional or post-transcriptional level (Fig. 2). 


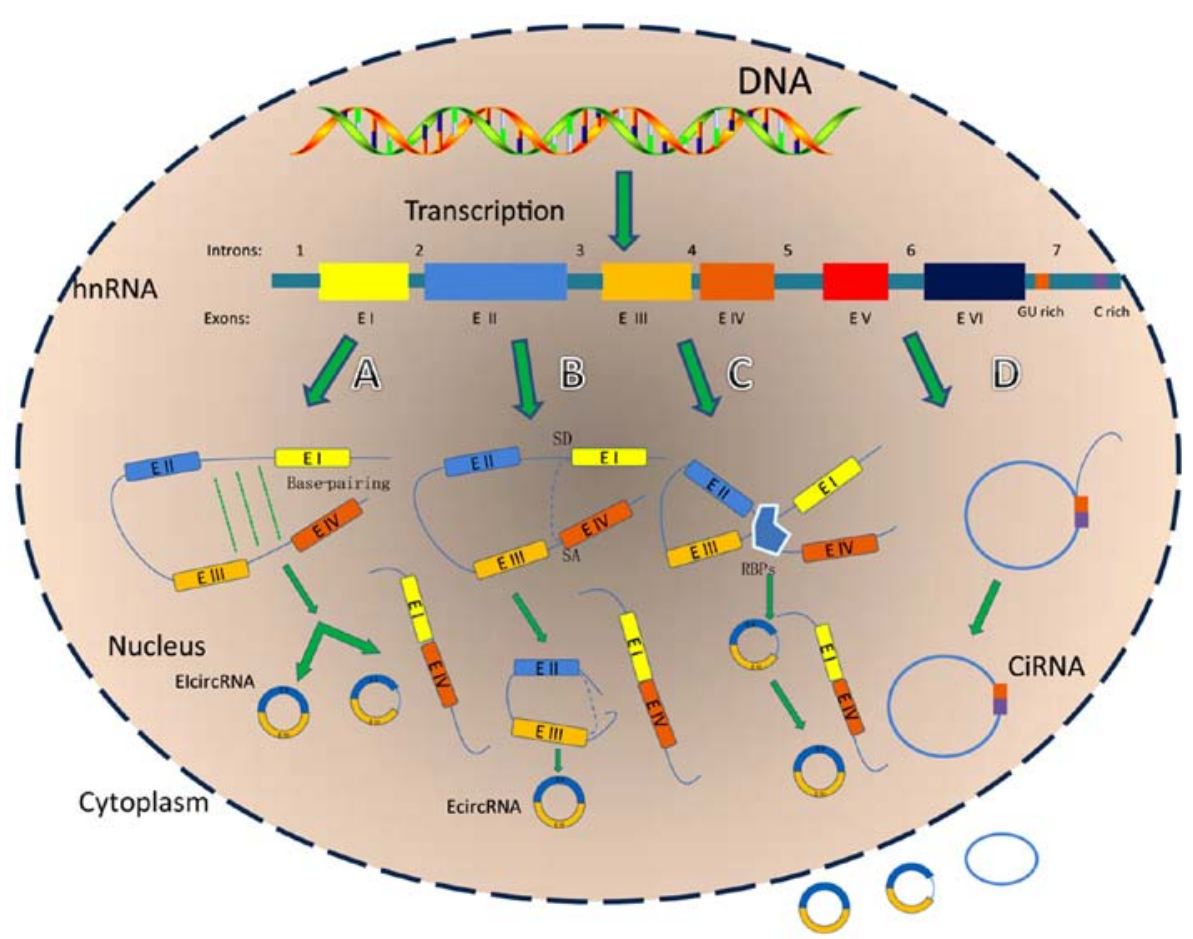

Figure 1.The mechanism underlying the synthesis of four types of circRNAs. In the nucleus of eukaryotic cells, DNA is transcribed to form hnRNA or pre-mRNA, which contain coding exons and introns. Based on the above products, circRNAs are generated via different splicing methods. (A) Intron-pairing-driven circularization. Two complementary introns form a circular structure containing several introns and exons through a base-pairing connection. Finally, introns are removed to form EcircRNAs, which can also lead to the formation of EIciRNAs. (B) Lariat-driven circularization. The exon 1 at the 3' end provides an SD site and the $5^{\prime}$ front end of exon 4 provides a covalent splice acceptor site after exon skipping. Then, a lasso-circular structure containing exons 2 and 3 forms. EcircRNAs are produced when introns are removed. (C) RBP-driven circularization. The binding of RBPs acts as a vehicle that binds two non-adjacent introns. Then circRNAs are generated after the removal of introns. (D) ciRNAs. Introns form a lasso modality after splicing that is removed following dissociation, thereby forming stable circular loops via binding with the 5-splice site (orange box), which is rich in ' $\mathrm{C}$ ' residues and the branch point (purple box) of 'GU-rich' sequences; this generates the ciRNAs after debranching. The majority of the aforementioned circRNAs are produced in the nucleus. Due to their unique circular structure they can enter the cytoplasm, as well as the intercellular substance and blood through exosomes, which are extremely stable and are not easily degraded by RNA nucleases. hnRNA, heterogeneous nuclear RNA; circRNA, circular RNA; ciRNA, circular intronic RNA; pre-mRNA, precursor messenger RNA; EcircRNA, exonic circular RNA; EIcircRNA, exon-intron circular RNA; SD, splice donor; RBP, RNA-binding protein.

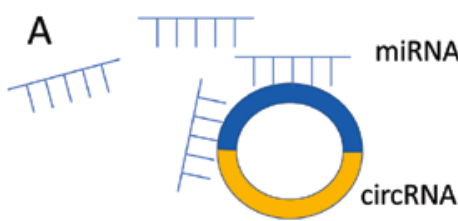

B
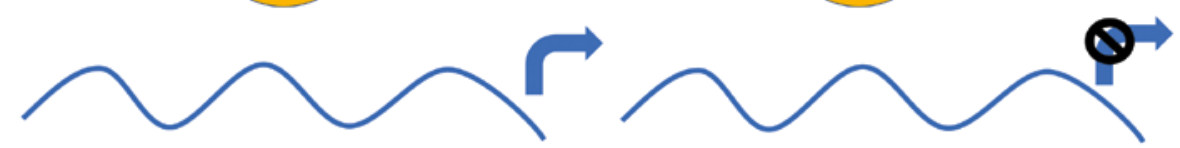

C

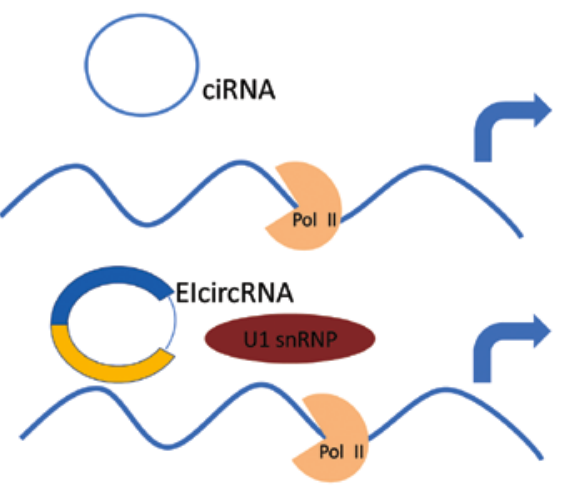

D

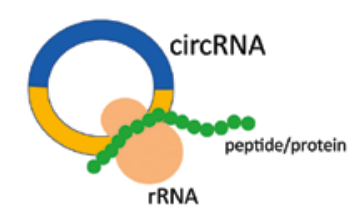

Figure 2. Four biofunctions of circRNAs. (A) CircRNAs can bind miRNAs and act as a sponge to regulate downstream transcription. (B) Through cis- and trans-acting effects, circRNAs can directly regulate transcription. (C) CiRNAs can enhance the expression of host genes by improving the activity of Pol II in the nuclei. EIcircRNAs can also interact with U1 snRNP and Pol II to enhance the host gene transcription in a cis-acting manner. (D) Part of the circRNA can also encode peptides or proteins. circRNA, circular RNA; ciRNA, circular intronic RNAs; miRNA, microRNA; Pol, polymerase; EIcircRNA, exon-intron circRNA; snRNP, small nuclear ribonucleoproteins. 
CircRNAs act as DNA replication regulators. DNA replication is a process by which two copies of DNA molecules are duplicated from the original template. During DNA replication, circRNAs are widely distributed in the nuclei, and can be paired with DNA to form DNA-RNA triple helixes, which affect DNA replication as they bind with the corresponding DNA strand. Recently, Schmitz et al (45) reported that pRNA can interact with transcription termination factor-I, a target transcription factor, to form a DNA-RNA triplex and consequently recruit DNA methyltransferase $3 \beta$ to inhibit ribosomal RNA expression. Additionally, lnc-Ras association domain family member 1 (RASSF1) binds with DNA to form a DNA/RNA complex at the transcription initiation site in order to inhibit the transcription of RASSF1A (46). Previous evidence indicates that the interactions between DNA and nucleic circRNAs may be similar to those of linear ncRNAs (47).

CircRNAs act as miRNA sponges. miRNAs are a class of short $(\sim 22 \mathrm{nt})$ and common ncRNAs that regulate gene expression after transcription via the direct base pairing of miRNA target sites (48). As regulators of competitive endogenous RNAs (ceRNAs), circRNAs can negatively regulate the activity of miRNAs by competing with mRNA-miRNA binding (49). For example, ciRS-7 [the circRNA sponge for miR-7, used in the transcription of human cerebellar degeneration-related protein 1 (CDR1)] contains over 70 selectively preserved miRNA target sites. CiRS-7 is derived from the antisense transcript of the CDR1 gene and can be endonucleolytically cleaved at an miR-7 target site via close binding with miR-671 in an Argonaute2 (AGO2)-dependent manner. Therefore, due to lysing of ciRS-7, miR-7 can be freed. It has been identified that the increase in the expression of miRNA target genes is as a result of the overexpression of CDR1/ciRS-7. Furthermore, SRY and testis specific circRNAs could act as an miR-138 sponge to regulate gene expression (12). Currently, serving as a miRNA sponge is thought to be the major function of certain circRNAs.

CircRNAs act as gene expression regulators. Previous studies have indicated that circRNAs may serve key roles in gene expression regulation $(13,50,51)$. Zhang et al (27) and Memczak et al (13) demonstrated that circRNAs could regulate parental gene expression, and that circRNAs are widely detected in the nuclei with a small concentration on miRNA targets; most notably, inhibition of ciRNAs may have led to the reduced expression of their parental genes. Moreover, in-depth studies have revealed that EIciRNAs, circ-eukaryotic translation initiation factor 3 subunit $J$ and circ-poly (A) binding protein interacting protein 2 , are primarily distributed in the nuclei, interacting with the $\mathrm{U} 1$ small nuclear ribonucleoprotein particle and RNA Pol II to enhance parental gene transcription in a cis-acting manner (38). In addition, a ciRNA, such as ci-ankyrin repeat domain 52 (ankrd52), could interact with elongation Pol II machinery and actively regulate Pol II transcriptional activity via enrichment of the transcription sites of parental genes. Thus, knockout of ci-ankrd52 may reduce parental gene expression (27).

From these results, we hypothesize that for circRNAs containing intronic sequences, namely EIciRNAs and ciRNAs, transcriptional activation may be their general function; in addition, their regulatory functions likely explain the rich existence of EIciRNAs and ciRNAs in the cell nuclei.

CircRNAs act as RBP sponges. RBPs participate in multiple biological activities, including cell proliferation, motility, apoptosis, senescence and cell responses to oxidative stress via posttranscriptional regulation such as RNA alternative splicing, conservation, transport and translation $(52,53)$. Previous studies have demonstrated that circRNAs can act as sponges for RBPs by stably binding with trinucleotide repeat containing 6A (18), RNA quaking homolog KH domain containing RNA binding (19), mannose binding lectin (33), AGO proteins (12), Pol II (54), and eukaryotic initiation factor 4A-III (55), to form RNA-protein complexes (RPCs). These RPCs may control the library of RBPs or small RNAs, and then exert mutual effects with their linear RNA counterparts (29). In addition, CircInteractome, a new online tool to analyze RBP binding sites on human circRNAs, can predict the potential circRNAs binding sites for miRNAs for a given RBP (56). For instance, hsa_circ_0024707 has 85 predicted positions as a sponge for AGO2, and the relatively mature hsa_circ_0000020 contains some RBP binding sites, such as circ-fragile $\mathrm{X}$ mental retardation 1 which contains 10 sites and circ-ELAV like RNA binding protein 1 which has 6 sites (56).

CircRNAs act as translation product translators. As a number of circRNAs can bind open reading frames (ORFs), one hypothesis is that they can be translated as protein fragments. Perriman and Ares (57) revealed that a simple green fluorescent protein ORF in a circRNA could be expressed directly in Colibacillus. In addition, a circRNA (220 nt) of a virus related to rice yellow spot virus that could code for a $16-\mathrm{kDa}$ protein was discovered by AbouHaidar et al (58). It has also been reported that protein/polypeptides can be coded by circRNAs both in vitro (59) and in vivo (57); however, only when the RNAs contain prokaryotic ribosome-binding sites (57), or internal ribosome entry site elements (IRES) unlike their canonical counterparts. Recently, the circRNA database, circRNADb, which contains $>32,000$ human EcircRNAs, was established (60). It offers detailed information on the circRNAs, including their sequence, ORF and IRES for users to predict the translatability of certain circRNAs. However, there is no well-founded evidence to verify how EcircRNAs that are generated from spliceosomes can act as mRNAs.

In addition to acting as miRNA sponges and transcriptional regulators, circRNAs can also positively act as mRNA traps that regulate protein expression by isolating the translation initiation point (61). For the first time, Yang et al (62) recently reported that $\mathrm{N}^{6}$-methyladenosine $\left(\mathrm{m}^{6} \mathrm{~A}\right)$ is the most common and abundant base modification of RNA and promotes the protein translation of circRNAs in the human body. The group demonstrated that $\mathrm{m}^{6} \mathrm{~A}$ motifs contained many circRNAs and only one $\mathrm{m}^{6} \mathrm{~A}$ was required to drive translation initiation. Most importantly, translation of $\mathrm{m}^{6} \mathrm{~A}$-driven circRNAs was quite common. A large number of endogenous circRNAs have the potential for translation, indicating that they may have a regulatory role in the stimulation of circRNA-derived proteins in cell responses to environmental stressors. 


\section{Metabolism of circRNAs}

CircRNAs are highly stable due to their unique structure; however, the underlying mechanisms of cell degradation and/or detectable circRNAs have not been elucidated. A previous study suggested that during their experiments, when multiple circRNAs were secreted from three different types of cells, the cells may have removed circRNAs through vesicles, such as exosomes and microcapsules (63). This phenomenon indicated that circRNA concentrations may exceed their linear counterparts during extracellular vesicle (EV) preparation when compared to the cell, implying that the mechanism underlying the reduction in circRNAs may be via EV release from the cells into the extracellular space.

\section{Public databases on circRNAs}

To perform classification of circRNAs, the present review evaluated the current online databases (Table I). These databases describe circRNAs from published articles and GenBank annotations. They list circRNAs that have been experimentally confirmed, those that are computational predictions and those that are based on the ORF predicted size. Herein, we list several key features of the databases.

i) Circbase: Basic information analysis of circRNAs. The latest version of the database was released in June 2017, and it can assist with gaining a specific understanding of the screening and verification of circRNAs; it is also possible to directly download relevant sequence and annotation information (64). ii) CircNet: ceRNA functional research database. Using 464 RNA-seq sequencing datasets, new circRNA predictions and genome annotations were performed, and known and newly predicted circRNA expression was calculated to construct a circRNA-miRNA-gene regulatory network from which the above information was obtained (65). iii) Circ2Traits: A circRNA database that generates potential associations with human diseases or traits to construct an interaction network by predicting the interactions between miRNAs and protein-coding genes, long non-coding genes, and circRNAs, thereby constructing an interactive network for Gene Ontology enrichment analysis of protein-coding genes in miRNAs-circRNA interaction groups (54). iv) CircRNADb: circRNA-encoded protein database. A total of 32,914 human exon circRNA records were included, each of which included genomic location information, RNA editing, corresponding genomic sequences, IRES sequence elements, predicted ORFs, and the related references (60). v) CircInteractome: A predictive binding target database. The binding sites of the known 109 RNA binding proteins to circRNAs in circbase were predicted and the potential binding sites for miRNAs to circRNA were predicted using Targetscan software. This software also performs circRNA molecular searches, circRNA binding protein prediction, polymerase chain reaction (PCR) primer design and small interfering (si)RNA interference sequence design. It also displays the RBP binding site of circRNA, the possibility of analyzing circRNA as an RBP protein 'sponge' or 'decoy', and the RBP protein information that may be bound by the junction and flanking sequences of the circRNA (56). vi) CircBank: A total of 140,790 human circRNAs were recorded in the circBank database, and each
circRNA record contains detailed information. The information for each circRNA mainly includes: The detailed sequence of the circRNA, the more homologous circRNA and its corresponding sequence in mice, the predictive analysis of miRNA binding, the ORF predictive information, the mutations and polymorphisms recorded by COSMIC Site summary, and the $\mathrm{m}^{6} \mathrm{~A}$ modification information (64). Notwithstanding the cancer-associated expression datasets, we expect the circRNA expression database, as displayed in Table I, to merge with other datasets to become more disease-oriented.

\section{CircRNAs and human disease}

Based on the pivotal role of genetic regulation, the altered expression of circRNAs associated with specific diseases is beginning to be increasingly reported. Altered circRNA expression has been observed in several diseases including diabetes, atherosclerosis, heart disease and neurological disorders (Table II).

\section{CircRNAs and cancer}

Recent studies have suggested that circRNAs may have a pivotal role during the development and progression of different types of cancers (66-68). CircRNAs have become a novel area of interest in the early diagnosis and therapy of cancers due to their abundance, high stability and notable regulatory functions. The fact that circRNAs appear to behave as miRNA sponges has also increased this interest (69). The expression of an miRNA is dysregulated via different mechanisms in humans, such as miRNA gene amplification or deletion, the abnormal transcriptional regulation of miRNAs, and epigenetic changes in miRNA generation (70). Zheng et al (71) analyzed a total of six human normal tissues (brain, heart, lung, liver, colon, and stomach) and seven human cancers (bladder cancer, breast cancer, hepatocellular carcinoma, gastric cancer, colorectal cancer, renal clear cell and prostate adenocarcinoma) to obtain the sequencing data of over 27,000 circRNA candidate genes. Compared to normal tissues, circRNAs were usually downregulated in tumor tissues and circRNA levels were significantly associated with clinical features including staging, age, gender and distant metastasis, due to errors in the back-splicing machinery of malignant tumors, the dysregulation of miRNAs due to the degradation of circRNAs in cancerous tissues, or the reduction in circRNAs as a result of increasing cell proliferation (72). In addition, circRNAs are observed widely in exosomes (73), illustrating that a potential future strategy to diagnose cancer may involve detecting blood plasma circRNAs. Herein, we discuss the latest discoveries focusing on abnormal circRNAs in cancer (Table III; Fig. 3).

CircRNAs and gastric cancer. Via RNA-seq analysis, 180 circRNAs have been detected with diverse expression in gastric tumor tissues as differentiated from normal tissues (74). Hsa_circ_002059, a representative circRNA, was observed to be downregulated in 101 gastric cancer tissues (73). The dysregulated expression of hsa_circ_002059 was markedly correlated with sex, age of disease onset, tumor-node-metastasis (TNM) stage and distant metastasis (73). In conclusion, these results 


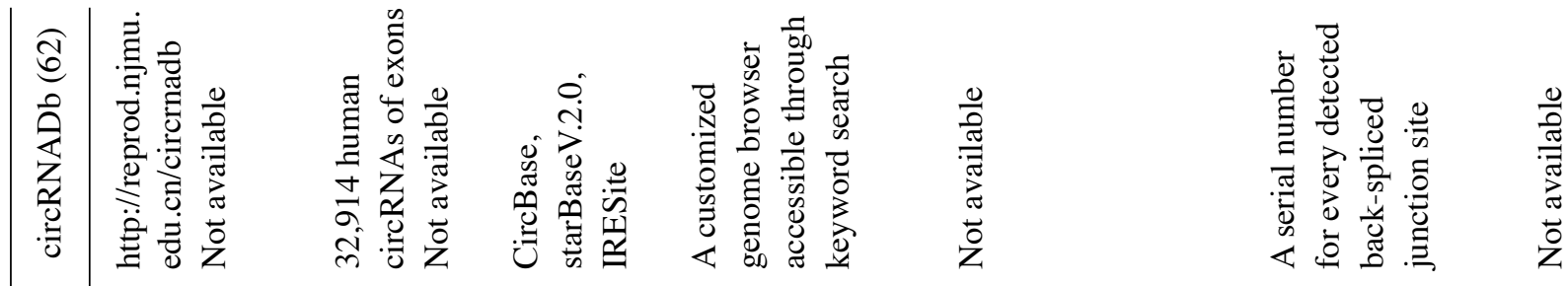

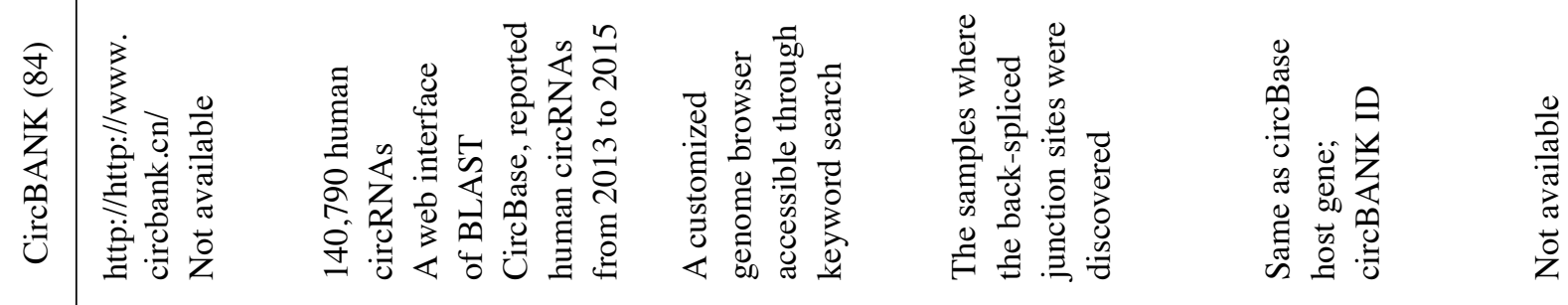

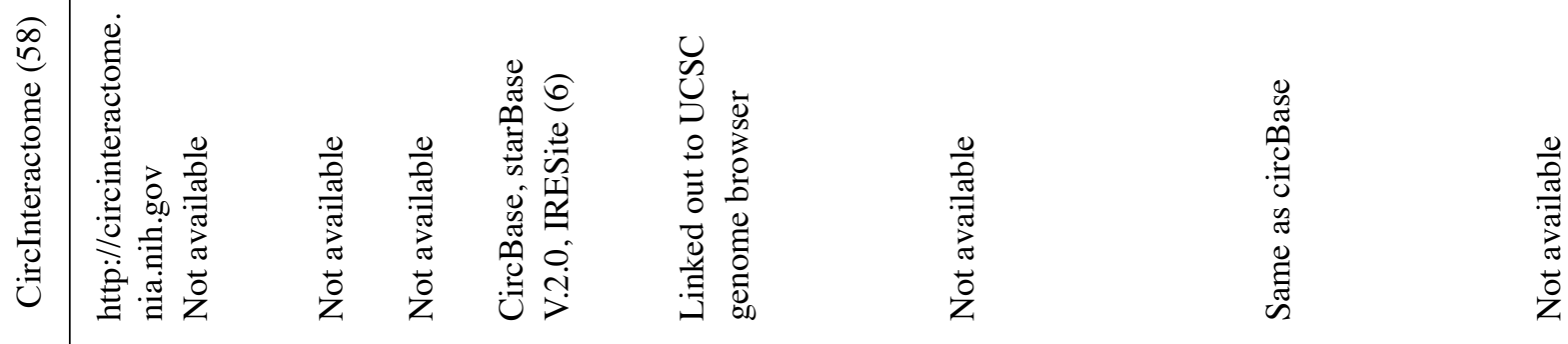

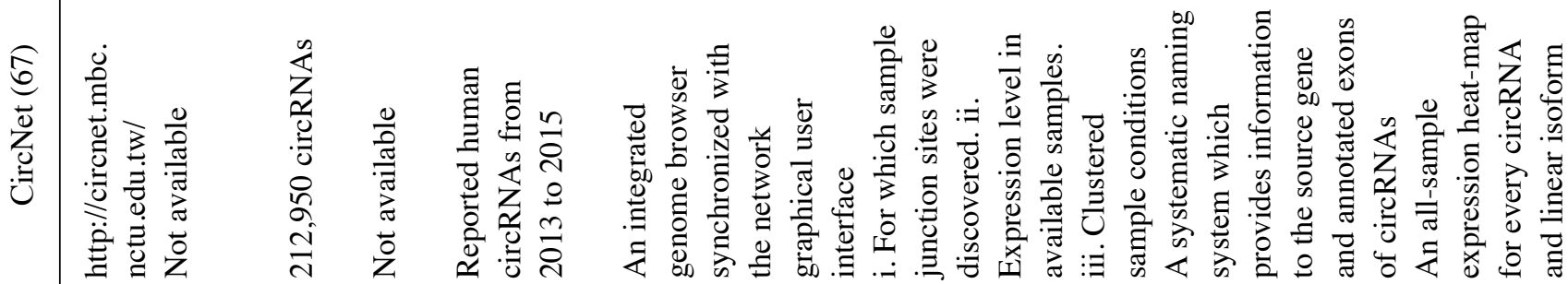

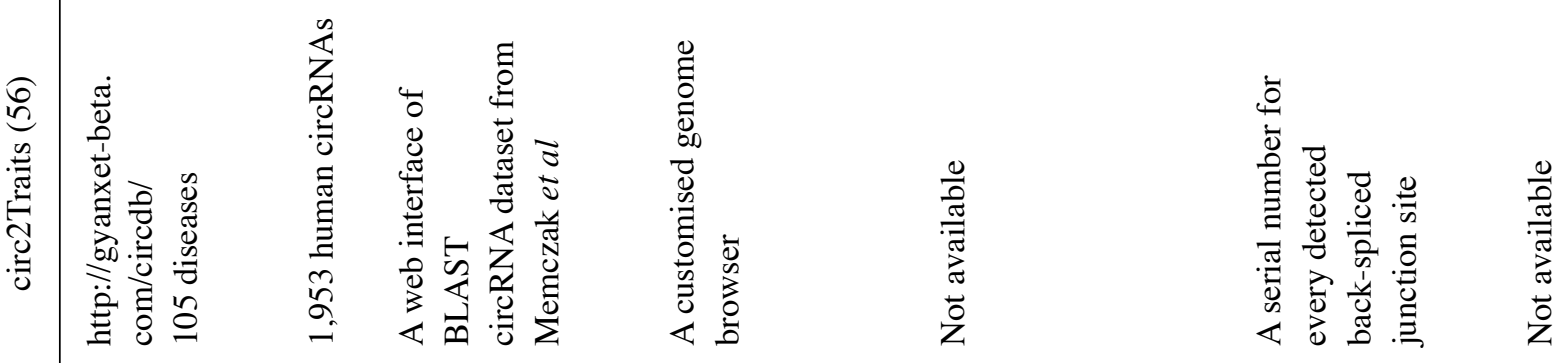

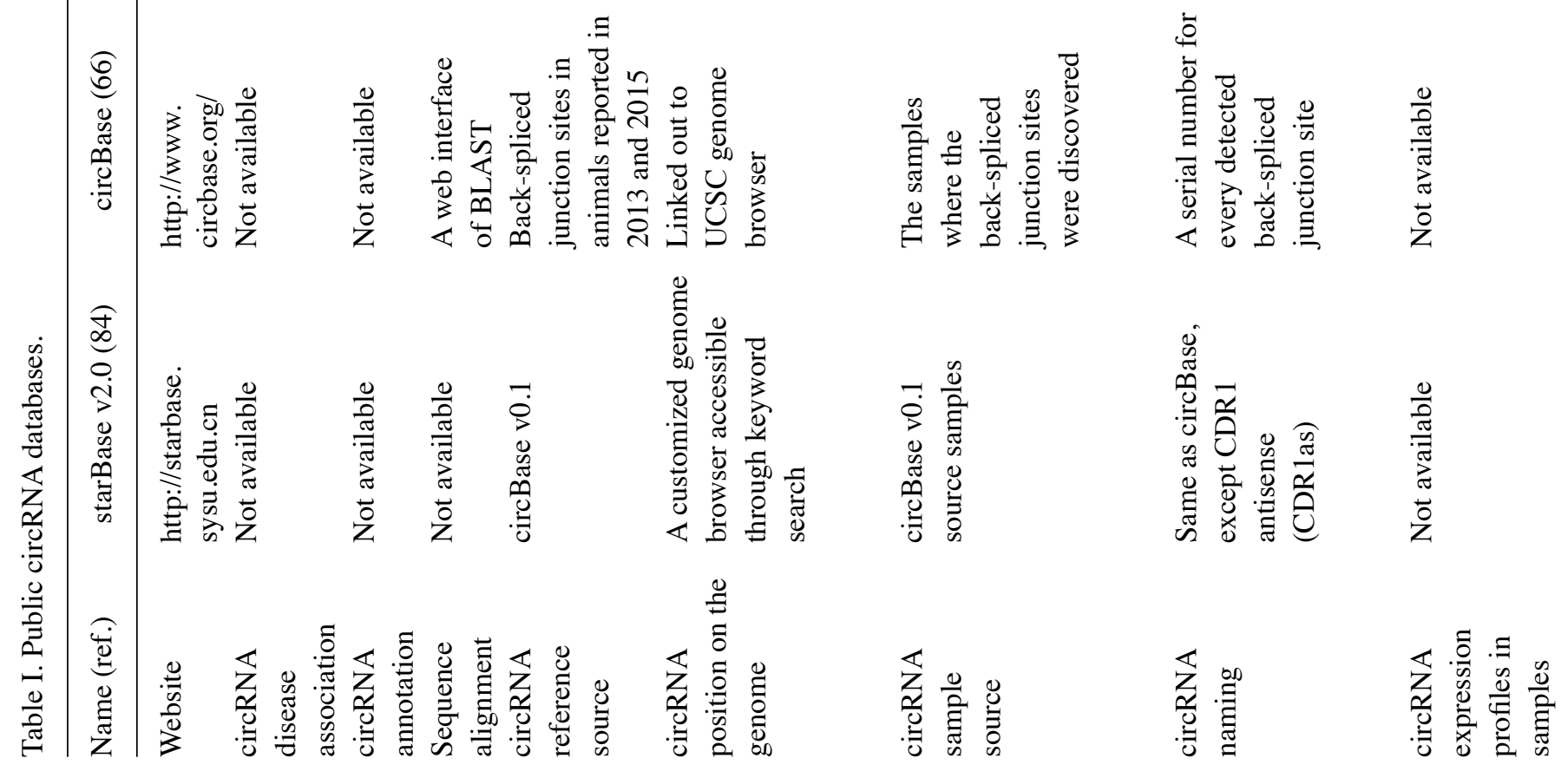


suggest that circRNAs, particularly hsa_circ_002059, may be potentially stable biomarkers for gastric carcinoma diagnosis and staging.

Another circRNA, circPVT1, has also been confirmed to be upregulated in gastric cancer (74). In addition, circPVT1 was found to suppress miR-125, which is a tumor suppressor family sponge. This circRNA also indirectly regulated the expression of the transcription factor, E2F transcription factor $2(\mathrm{E} 2 \mathrm{~F} 2)$, as determined by a luciferase reporter assay, and was found to serve a crucial role in the progression of the cell cycle and tumor inhibitor protein functions (74). Therefore, the abnormal expression of circPVT1 may reduce the anticancer effect of E2F2 and miR-125b. Furthermore, circPVT1 was shown to facilitate the colony formation of gastric cancer by inhibiting miR-125 (74).

Expression of the circRNA, hsa_circ_0000096, also called circHIAT1, has also been confirmed to be downregulated in gastric cancer cell lines and cancerous gastric tissues when compared with non-cancerous epithelial cells and normal gastric tissues (75). Furthermore, the knockdown of hsa_circ_0000096 by siRNA has been observed to significantly inhibit cell proliferation and migration both in vitro and in vivo, and it also was found to suppress the progression of the cell cycle, arresting the $\mathrm{S}$ phase of cell transition from G0/G1 in gastric cancer cells (75). In addition, knockdown of hsa_circ_0000096 has been observed to suppress tumor growth in xenograft nude mouse models. It is commonly thought that a high expression of circRNAs may be observed in cancers; however, tumor-inhibiting genes have been reported in low levels. Nevertheless, hsa_circ_0000096 seems to be an exception to the above. This phenomenon could be explained by its interaction with miRNAs. The circRNA databases have revealed that hsa_circ_0000096 can bind with 17 different miRNAs. More, previous results of reverse transcription-quantitative PCR (RT-qPCR) have confirmed that the cluster of differentiation- 40 regulator decreases the levels of miR-224 in a number of gastric cancer cell lines and the primary function of miR-200a is to increase E-cadherin levels, following the knockdown of hsa_circ_0000096 (75).

These previous studies have presented the clinical implications of hsa_circ_002059 and hsa_circ_0000096 as biomarkers, as determing their expression level is able to distinguish between normal gastric mucosa and gastric cancer tissue. In addition, these circRNAs can also be used as molecular biomarkers to evaluate gastrectomy.

CircRNAs and lung cancer. The circRNA circ-itchy E3 ubiquitin protein ligase (ITCH) has been confirmed to play a crucial role in suppressing lung cancer progression by functioning as a miRNA sponge in multiple carcinogenic miRNAs. The expression of ITCH can be enhanced by this action and in turn inhibit the Wnt/ $\beta$-catenin signaling, which is a significant pathway involved in tumor cell proliferation and the cell migration process. Previous studies have verified that circ-ITCH can bind to different types of miRNAs, including, miR-7, miR-17, miR-128, miR-214, and miR-216b $(12,13,76)$. Wan et al (77) reported that circ-ITCH expression was greater in cancerous tissues when compared with that noted in adjacent non-cancerous tissue and normal cells of 78 matched patients with lung cancer. The report demonstrated that $>70 \%$ 
Table II. circRNAs identified in human diseases.

\begin{tabular}{|c|c|c|c|}
\hline Disease & circRNAs & Function & (Refs.) \\
\hline \multirow[t]{3}{*}{$\begin{array}{l}\text { Ischaemic heart } \\
\text { Disease (IHD) }\end{array}$} & cANRIL & $\begin{array}{l}\text { An increased risk of atherosclerosis by repressing of the } \\
\text { INK4A/ARF locus }\end{array}$ & $(49)$ \\
\hline & cZNF292 & Regulation of hypoxia in endothelial cells and control of angiogenesis & $(12)$ \\
\hline & hsa_circ_0124644 & Upregulated in coronary artery disease & $(52)$ \\
\hline \multirow[t]{2}{*}{ Alzheimer's disease } & ciRS-7 & Upregulated UBE2A that aids the clearance of amyloid peptides & $(54)$ \\
\hline & circPVT1 & Cell senescence inhibitor & $(58)$ \\
\hline Diabetes & ciRS-7 & $\begin{array}{l}\text { Inhibits miR-7 function in islet } \beta \text { cells, which can inversely improve } \\
\text { secretion insulin }\end{array}$ & (59) \\
\hline
\end{tabular}

circRNAs, circular RNAs.

of the lung cancer tissues had significantly decreased expression of circ-ITCH (77). To elucidate the associations between circ-ITCH and the miRNAs, miR-7 and miR-214, circ-ITCH plasmids were cotransfected into the human lung cancer cell lines A549 and H460, and a luciferase reporter of miR-7 or miR-214 was established; the luciferase activity was then detected. After transfection with an empty vector in both cell lines (including the control group containing plasmid with circ-ITCH), the luciferase activity was significantly reduced in a concentration-dependent manner by miR-7 or miR-214 mimics. However, no obvious change in luciferase activity was observed in cells with circ-ITCH overexpression. Therefore, these results indicated that circ-ITCH may suppress miR-7 and miR-214 by binding to them as a sponge in lung cancer tissues (77).

Yao et al (78) confirmed that in tissues from 101 patients with non-small cell lung cancer (NSCLC), circRNA_100876 was significantly upregulated when compared with the corresponding adjacent normal lung tissues. Among the estimated clinicopathological results, the overexpression of circRNA_100876 was significantly associated with regional lymph node metastasis and advanced stages of the tumor. Patients with NSCLC and elevated circRNA_100876 expression exhibited notably shorter overall survival than those with low expression (78). This therefore suggested that circ_100876 may be a potential biomarker for tumor cell proliferation, progression and metastasis in NSCLC (78).

CircRNAs and colorectal cancer (CRC). As aforementioned in lung cancer, circ-ITCH was also observed to have reduced expression in 45 matched colorectal cancer (CRC) tissues when compared with that noted in adjacent non-cancerous colorectal tissues (76). In a previous study investigating the CRC cell lines, SW480 and HCT116, circ-ITCH sponge activity was demonstrated for miR-7, miR-20a, and miR-214 (76). These miRNAs have been revealed to downregulate many target genes that are primarily involved in the regulation and completion of the G1/S transitional phase, including the proliferative target gene cyclin D1 (76). It has also been suggested that the aberrant expression of circ-ITCH may inhibit the expression of the target genes c-Myc and cyclin D1 of the Wnt/ $\beta$-catenin signaling pathway (76). These results indicated that circ-ITCH may be involved in the regulation of the $\mathrm{Wnt} / \beta$-catenin signaling pathway, and in turn, the Wnt/ $\beta$-catenin signaling pathway may serve a significant regulatory role in cell proliferation and migration (76). Consistent with this, overexpression of circ-ITCH was found to reduce HCT116 and SW480 cell proliferation. Consequently, circ-ITCH is likely to have an anti-proliferative role in CRCs (76).

Another circRNA, hsa_circ_001569, has also been demonstrated to exhibit high levels of expression in CRC when compared with that noted in non-cancerous tissues $(54,79,80)$. miR-145 could bind hsa_circ_001569, which contains three target genes [E2F5, B-cell lymphoma 2 associated athanogene 4 (BAG4) and formin like 2 (FMNL2)], and may be inhibited by the 3,-untranslated region promoter, as determined by evaluating two public databases (StarBase v2.0 and circBase) and three bioinformatic websites (TargetScan, Pictar and miRANDA) $(54,64,81)$. A previous study reported that E2F5 is a transcription factor that can control the gene expression of proteins involved in cell cycle control (82), that BAG4 is involved in cancer cell invasion $(83,84)$, and that FMNL2 participates in cell growth, motility, invasion, metastasis and epithelial-mesenchymal transition (EMT) $(85,86)$. hsa_circ_001569 has been demonstrated to promote CRC cell proliferation and invasion by blocking the downregulation of E2F5/BAG4/FMNL2 by miR-145 (80). Subsequently, the group revealed that hsa_circ_001569 promotes cell proliferation by increasing the number of $\mathrm{S}$ and $\mathrm{G} 2 / \mathrm{M}$ phase cells in the cell cycle (80). Furthermore, hsa_circ_001569 was knocked down in SW620 and LoVo cells, which has been indicated to reverse invasion (80). Notably, unlike the other circRNAs that have been mentioned, hsa_circ_001569 may directly inhibit the transcriptional activity of miR-145, rather than inhibiting its expression (80).

Similarly, CRC tissues have also been revealed to have high levels of circ-BTG3 associated nuclear protein (BANP) (87). Knockout of circ-BANP using siRNA also reduced the proliferation of the CRC cell lines HCT116 and HT29. Furthermore, the expression of phosphorylated (p)-protein kinase B (Akt) was downregulated via this knockout, indicating that it may be involved in the phosphoinositide-3 kinase (PI3K)/Akt signaling pathway, which is known to have roles in controlling cancer cell survival and the cell cycle (87). 


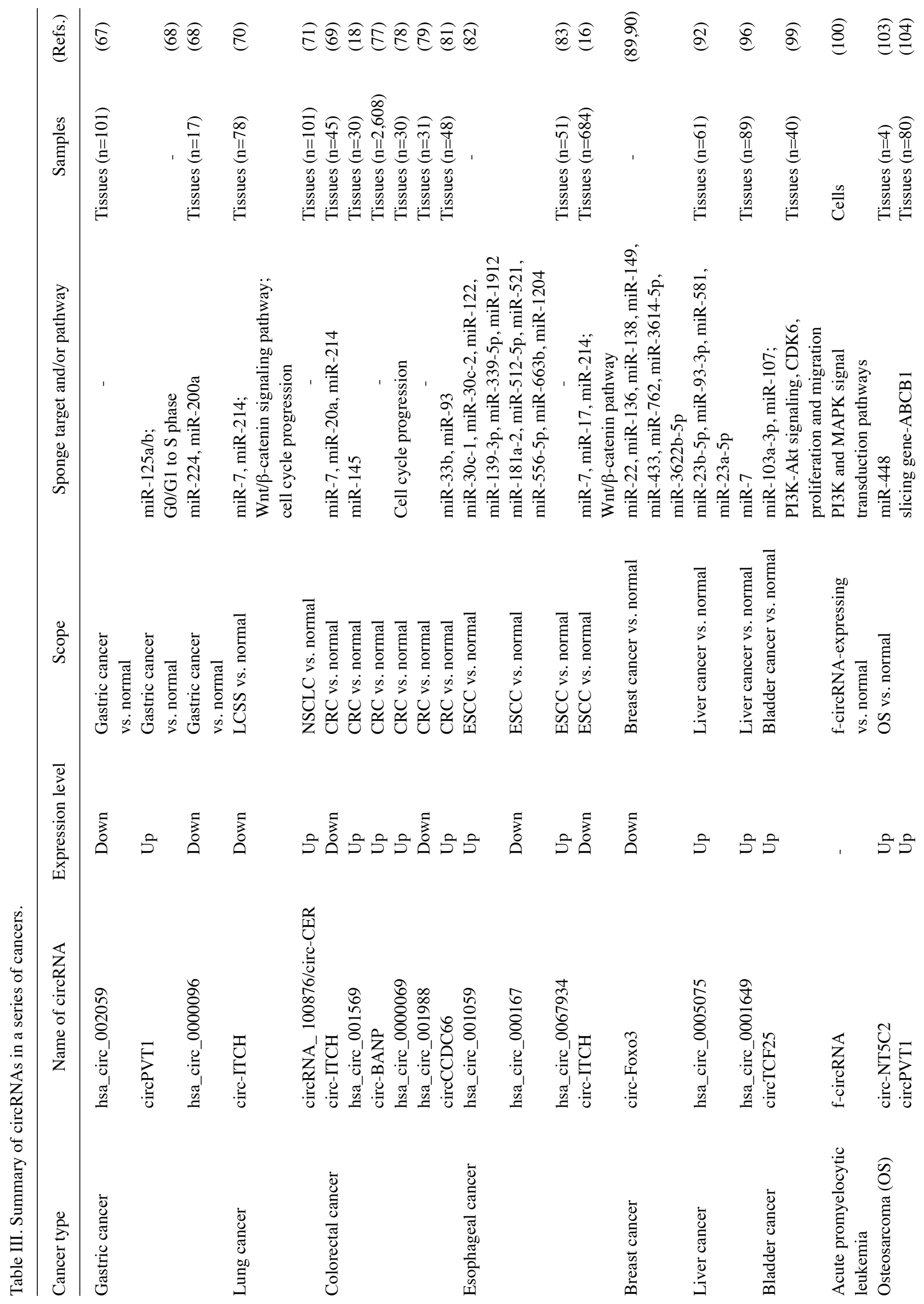


Guo et al (88) demonstrated that hsa_circ_0000069 was also overexpressed in CRC, as determined by unsupervised hierarchical cluster analysis. Inhibition of hsa_circ_0000069 with siRNA may suppress cell proliferation, migration and invasion, as well as induce HT-29 cell arrest at the G0/G1 phase of the cell cycle (88).

Hsa_circ_001988 was also found to be downregulated in CRC cell lines when compared with normal samples in 31 matched CRC tissues and non-cancerous colon mucosa (89). In addition, the results indicated that the expression level of hsa_circ_001988 was associated with cancer cell differentiation, and even perineural invasion. Nerve infiltration is a clear predictor of the prognosis of CRC patients, which is related to the survival time and recurrence of CRC $(71,90)$. These results indicate that hsa_circ_001988 may be a potential new biomarker of CRC prognosis (89). Hsiao et al (91) analyzed the expression of circRNAs and adjacent non-tumor tissues in 48 colorectal tumors. The results revealed that circ-cyclin B1, circ-cyclin-dependent kinase (CDK)-13, and circ-coiled-coil domain containing 66 (CCDC66), which is comprised of exons 8-10, were elevated in CRC tissues (91). Notably, the RT-qPCR results from multiple cancer cell lines, including colorectal (Caco-2, HCT116, HT-29, and LS123), mammary gland (MCF-7, MDA-MB-231, and MDA-MB-468), pancreatic (BxPC-3 and MIA PaCa-2) and cervical cancer (HeLa) cell lines, demonstrated that there was an abnormal expression of circCCDC66, which suggested that this circRNA may have important implications for the majority of oncogenes (91). In addition, circCCDC66 is thought to regulate a variety of pathological processes, including cell proliferation, migration and invasion in vivo and in vitro (91). Furthermore, a previous study identified 99 potential miRNAs that could bind exons 8-10 of circCCDC66. This evidence suggests that circCCDC66 may act as an miR sponge to protect the miRNA activity of the oncogene, Myc, from CRC. It's key role in CRC was confirmed in miR-33b and miR-93 by knocking out circCCDC66 (91).

CircRNAs and esophageal cancer. Previous studies have reported that several circRNAs, including hsa_circ_001059, hsa_circ_000167, hsa_circ_0067934, and circ-ITCH, may be involved in cancer-associated mortality in esophageal squamous cell carcinoma (ESCC) (92-94). Su et al (93) identified two distinct circRNAs, hsa_circ_001059 and hsa_circ_000167, from $>3,700$ human circRNAs, that were observed with altered expression levels in the human anti-radioresistant esophageal cancer cell line KYSE-150R, when compared with the adjacent normal cell line KYSE-150. CircRNA-miRNA coexpression network analysis indicated that the two circRNAs were the key factors in the potential circRNA-miRNA network (93). These results indicated that circRNAs may have dysregulated expression in esophageal cancer cells during the development of radiation-resistant expression (93).

The circRNA hsa_circ_0067934 has also been examined in ESCC tissues and was also significantly overexpressed; it is thought to be associated with differentiation, as well $\mathrm{T}$ stage I-II and TNM stage I-II (94). Furthermore, siRNA knockdown of hsa_circ_0067934 activity was reported to inhibit cell proliferation, migration, and invasion in ESCC cells (94). When considering other clinical factors, lymph node metastasis or tumor size was not associated with the 


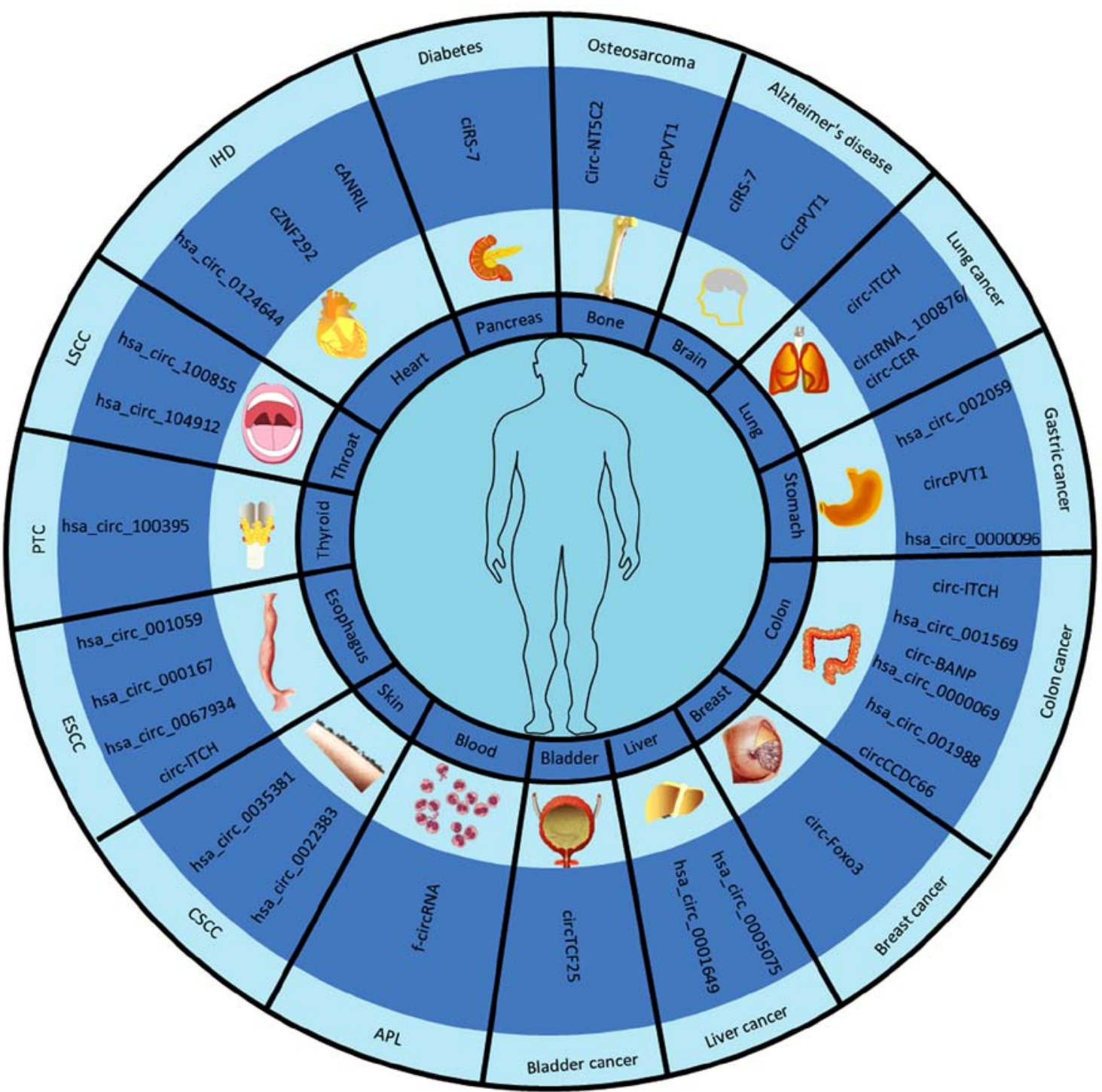

Figure 3. Functional circRNAs summarized in different diseases. The map presented exhibits the circRNAs that have been reported in different diseases, including various types of cancer. circRNA, circular RNA; IHD, ischaemic heart disease; LSCC, laryngeal squamous cell cancer; PTC, papillary thyroid cancer; ESCC, esophageal squamous cell carcinoma; cSCC, cutaneous squamous cell carcinoma; APL, acute promyelocytic leukemia

expression of hsa_circ_0067934. Notably, TNM staging was applied to assess patient prognosis, and it is possible that hsa_circ_0067934 may also have the potential to become a biomarker for diagnosing ESCC.

In addition, following analysis of 684 ESCCs and adjacent non-cancerous tissues, circ-ITCH was shown to be downregulated in ESCC, acting as a tumor inhibitor gene for lung cancer and CRC (92). Li et al (92) revealed that circ-ITCH can act as a sponge for miR-7, miR-17, and miR-214, passively regulating the Wnt/ $/$-catenin pathway via the upregulation of the target gene ITCH. Therefore, this result indicates that circ-ITCH may be a passive regulator of the $\mathrm{Wnt} / \beta$-catenin signaling pathway via the indirect sponge effect of miRNAs (92).

CircRNAs and breast cancer. Approximately one fifth of breast cancer cases worldwide are diagnosed as ductal carcinoma in situ (DCIS) via mammography (95). Generally, DCIS is deemed to be highly curable; however, some women with DCIS unfortunately suffer from the life-threatening type of invasive breast cancer, invasive ductal cancer (IDC); even though the invasive factors of progression remain unclear. This may result in the same histological subtypes sharing similar patterns of gene expression $(96,97)$.

Galasso et al (97) analyzed the expression of circRNAs in the DCIS/IDC samples of five patients and also evaluated the invasive breast cancer cell line, MCF-7, using published RNA-seq databases and the bioinformatics testing pipeline, CIRCexplorer. Hsa_circ_0122662 and hsa_circ_0001358 were both identified in the IDC samples and the MCF-7 cell line. hsa-circ-0001358 was found to be associated with five miRNAs, miR-200c-3p, miR-200b-3p, miR-376a-3p, miR-376b-3p and miR-429, as determined by the Starbase human Pan Cancer tool (97). miR-200c-3p, miR-200b-3p, and miR-429, which belong to the miR-200 family, were found to reduce the migration and invasion of breast cancer cells $(98,99)$. The genes known to participate in epithelial-mesenchymal transition (EMT) that are also known targets of the miR-200 family were detected, namely zinc finger E-box binding 
homeobox 1 (ZEB1), ZEB2, vimentin, B lymphoma Mo-MLV insertion region 1 homolog, and fibronectin 1 (97). However, no significant interactions were identified (97). It is also worth noting that excess circRNAs were observed to be expressed in DCIS or IDC, indicating measurable differences in dynamic expression. As a result, differential circRNA expression may warrant further exploration in order to further understand the molecular mechanisms of DCIS progression to IDC.

Circ-forkhead box O3 (Foxo3) has been shown to be downregulated in breast cancer cell lines and breast tumor tissues when compared with that noted in adjacent non-cancer cell lines or non-cancerous tissues (100). Overexpression of circ-Foxo3 was found to significantly reduce proliferation and cell survival in the breast cancer cell line, MDA-MB-231 (101). Du et al (101) injected MDA-MB-231 cells transfected with circ-Foxo3 subcutaneously in nude mice, demonstrating that circ-Foxo3 suppressed tumor growth. Subsequently, through tumor section TUNEL staining, circ-Foxo3-transfected tumor cells were observed to exhibit extensive cell death, suggesting that apoptosis occurred in the tumor. In addition, 25 additional binding sites were examined in circ-Foxo3 for eight miRNAs, including miR-22, miR-136, miR-138, miR-149, miR-433, miR-762, miR-3614-5p, and miR-3622b-5p (100). It was also revealed that when these miRNAs were transfected into MDA-MB-231 cells, they decreased cellular apoptosis. Therefore, these studies indicated that circ-Foxo3 may serve a crucial biological role as a miRNA sponge.

CircRNAs and hepatocellular carcinoma. An increasing body of evidence has revealed that circRNAs are associated with the development of hepatocellular carcinoma (HCC). By analyzing integrated bioinformatic data, many circRNAs have been demonstrated to be involved in the overexpression of miR-181a-3p and thus, the enzyme O(6)-methylguanine-DNA methyltransferase, which is known to be involved in DNA disruption, thereby suggesting a potential link between circRNAs and HCC progression through miRNA regulation (102). Shang et al (103) in a recent and extensive study identified that there are 61 distinctly expressed circRNAs in HCC tissues when compared with that noted in adjacent normal tissues. Of the three mentioned above that were further verified through RT-qPCR, hsa_circ_0005075 was significantly associated with a number of clinicopathological factors of HCC patients (103). Through pathway analysis and Gene Ontology of hsa_circ_0005075, it was revealed that this circRNA was strongly associated with cell adhesion, which is an important process in tumor cell proliferation and metastasis (103). Furthermore, larger liver cancer tumors were found to exhibit a higher expression level of hsa_circ_0005075 than smaller tumors, demonstrating that hsa_circ_0005075 may regulate tumor growth. Therefore, hsa_circ_0005075 is likely to be a promising biomarker for HCC (103). In addition, the four binding sites, miR-23b-5p, miR-93-3p, miR-581, and miR-23a-5p, for hsa_circ_0005075 were located within HCC tissues, as determined via the predictive program Arraystar (DNASTAR, Inc., Madison, WI, USA), which performs sequence variation analysis (103). Shang et al (103) also demonstrated that miR-23a-5p has the largest interaction network, following analysis of the hsa_circ_0005075 circRNA-miR-mRNA network. Interestingly, miR-23b-5p has been observed to be downregulated in gastric and esophageal cancer $(104,105)$. It has been hypothesized that hsa_circ_0005075 may act as a miRNA sponge to suppress the expression of miR-23b-5p in cancer (103). As described above, the expression of hsa_circ_0005075 was high in HCC tissues, thus it has been suggested that it may be involved in tumor progression. However, further study is required to elucidate the molecular mechanisms underlying the development of HCC and how hsa_circ_0005075 functions as a miRNA sponge.

Hsa_circ_0001649 has also been indicated to have decreased expression in HCC tissues, when compared with that observed in adjacent liver tissues (106). Following the inhibition of hsa_circ_0001649 using siRNA, the expression levels of the pro-metastatic matrix metalloproteinases (MMPs), MMP9, MMP10 and MMP13, were increased (106). These results demonstrated that the expression of hsa_circ_0001649 is negatively correlated with the metastasis of HCC and thus, could represent a potential marker of HCC prognosis (106).

In addition, ciRS-7 has also been indicated to be significantly upregulated in HCC tissues (107). HCC cell proliferation and invasion were significantly reduced potentially via the release of miR-7 (107,108). Thus, ciRS-7 may also be a miR-7 sponge, affecting the expression of miR-7-targeted genes through an indirect regulatory pathway.

CircRNAs and bladdercancer. Bladder cancer is one of the most commonly diagnosed malignancy of the genitourinary system and is also one of the most common causes of cancer-associated mortality in men (109). Recently, Zhong et al (110) reported the altered expression of six circRNAs via high throughput microarrays. Using this method, the authors revealed that circ-transcription factor 25 (TCF25), circ-protein tyrosine kinase 2, circ-zinc finger RNA binding protein, and circBC048201 were significantly upregulated, and the two circRNA circ-family with sequence similarity 169 member A and circ-tripartite motif-containing 24 were both downregulated in bladder cancer tissues when compared with paired non-cancerous tissues. Further study revealed that circTCF25 could bind with the miRs, miR-103a-3p and miR-107, within the PI3K-Akt signaling pathway and affect cancer progression (110). Abnormal overexpression of circTCF25 was revealed to facilitate the proliferation and migration of bladder cancer cell lines by inhibiting miR-103a-3p and miR-107 (110). Analysis of the miRNA sponge, circTCF25, revealed that the elevated protein levels of CDK6 negatively regulated oncogenic factors (110). These results demonstrated that the circTCF25-miR-103a-3p/miR-107-CDK6 network has an important role in the development of bladder cancer (110).

CircRNAs and acute promyelocytic leukemia. Guarnerio et al (111) demonstrated that cancer-associated chromosomal translocations result in the fusion of circRNAs (f-circRNAs), which are produced by the transcriptional exons of different genes that are affected post-translation. Subsequently, the group analyzed the acute promyelocytic leukemia (APL) cell line NB4 and evaluated the expression of f-circRNA (either f-circ-progesterone receptor or f-circM9). The results supported the concept that f-circRNA, coupled with other tumor irritants, may support the progression of leukemia 
in humans (111). Further research also demonstrated that circRNAs are widespread in human thrombocytes and are increased by 17- to 188-fold when compared with that in nucleated tissues. As circRNAs are degraded by exonucleases, their localization relative to that of linear RNAs can be used as a marker of mRNA abundance in the absence of transcription (112). A previous study revealed that neoplasm-cultured platelet levels could differ in cancer patients with $96 \%$ accuracy when compared with healthy groups, and the localization of the primary tumor could be accurately identified with $71 \%$ accuracy from six cancer types by mRNA sequencing (113). These results demonstrated that f-circRNAs and changes in the level of blood platelets may become potential markers for the diagnosis and treatment of tumors.

CircRNAs and osteosarcoma. The incidence of osteosarcoma remains high in adolescents and children, and the overall survival rate is remains low. Identifying novel early detection markers and therapeutic targets are required for the treatment of osteosarcoma. Liu et al revealed that circ-5'-nucleotidase cytosolic II (NT5C2) was abnormally expressed in osteosarcoma as determined by high-throughput analysis; it promoted tumor cell progression and metabolism by regulating miR-448 expression (114). In addition, a previous study revealed that downregulation of circ-PVT1 decreased the expression of the classical multidrug resistance-associated gene, ATP binding cassette subfamily B member 1, suggesting that circ-PVT1 may be more efficient than alkaline phosphatase for the diagnosis of osteosarcoma (115). Taken together, the results of these studies indicate that circ-NT5C2 and circ-PVT1 may become early diagnostic markers and treatment targets.

CircRNAs and other types of cancers. Numerous circRNAs have been implicated in a wide range of cancers, including gastric, lung, colorectal, esophageal, breast, hepatic, vesical cancers, and in APL as discussed above (Table III). However, it is clear that circRNAs are not limited to these types of cancers. Due to the rapid expansion of the research field, researchers are frequently reporting the important roles of circRNAs in different types of cancers.

A previous study identified circRNA expression patterns in pancreatic ductal adenocarcinoma (PDAC), using an aberrant microarray chip. The results indicated that circRNAs are involved in the growth and metastasis of PDAC (116). Sand et al (117) revealed that 322 circRNAs are expressed in cutaneous squamous cell carcinoma (cSCC) and a total of 1,603 MREs were determined in the differentially expressed circRNAs. The results demonstrated that circRNAs are expressed in cSCC and participate in the formation of cancer through the involvement of complementary MREs, which interfere with miRNA sequences in the epigenetic control of cSCC-associated miRNAs (117).

Using Arraystar Human circRNA Microarray, Peng et al (118) validated 88 upregulated circRNAs and 10 significantly downregulated circRNAs in papillary thyroid cancers (PTC), compared to matched metastatic normal thyroid samples. Using these dysregulated circRNAs and predicted MREs, a well-connected network map of circRNA-miR interactions was established using Cytoscape software. One of the downregulated circRNAs, hsa_circRNA_100395, showed potential for functioning with two miRNAs associated with cancers, miR-141-3p and miR-200a-3p (118). This result indicates that the hsa_circRNA_100395-miR-141-3p or hsa_ circRNA_100395-miR-200a-3p interaction may be involved in the nosogenesis of PTC tumors (118). However, this theory requires further analysis to be proven correct.

Analysis of laryngeal squamous cell cancers (LSCCs) revealed that hsa_circ_100855 and hsa_circ_104912, among the 698 identified aberrant circRNAs, were markedly upregulated and downregulated, respectively (119). The upregulation of hsa_circ_100855 was related with the T3-4 stage, cervical lymph node metastasis and the late clinical stage of LSCC, while the downregulation of hsa_circ_104912 was correlated with late stage, cervical lymph node metastasis and poor differentiation. The results of this previous study indicated that hsa_circ_100855 and hsa_circ_104912 may be potential neoteric biomarkers for the diagnosis and progression of LSCC (119).

\section{Conclusions}

Taken together, these results indicate that circRNAs as mediators may be involved in the occurrence and development of cancer. Particular circRNAs, such as circ-ITCH, may even play crucial roles in more than one type of cancer. Nevertheless, the majority of the previous studies mentioned above lack clear and convincing arguments to explain the molecular mechanisms of action, and thus, it is essential to obtain a deeper understanding of the implications of their interactions in cancer on a molecular level. Therefore, the clinical significance of circRNAs as novel clinical diagnostic, metastatic and prognostic biomarkers requires further study.

CircRNAs are a class of abundant ncRNAs that have been demonstrated to regulate gene expression through a variety of complicated mechanisms, and may also have the potential to encode proteins; however, the underlying mechanisms have not been completely elucidated. Nevertheless, circRNAs are becoming potentially important regulators of cellular cytopathophysiology and potential biomarkers for disease onset or progression. The current levels of knowledge on circRNAs are relatively new and limited; thus, further research is urgently needed to fully understand their functions and clinical potential.

\section{Perspectives}

Recently, research into circRNAs has focused on their biogenesis and functions as miRNA sponges and biomarkers. Although there have been several studies on circRNAs, their bioinformatic functions and related molecular mechanisms require further study. In fact, most circRNA functions are still largely unexplored, such as their specific expression during tumor cell development, localization and metabolism. CircRNAs have good potential as clinical diagnostic and prognostic biomarkers when compared with miRNAs and lncRNAs, due to their stable ring structure. Based on their location, there are three subclasses of circRNAs: circRNAs are located in the cytoplasm and ciRNAs and EIciRNAs are both nuclear $(7,13,27)$, implying that circRNAs may play epigenetic roles in cell biology. 
To date, there is no uniform nomenclature for circRNAs, which has caused confusion regarding the terminology. Although there are two sets of nomenclature rules, they both have their own advantages and disadvantages. One is named according to the nomenclature in the circBASE database, such as 'hsa_circRNA_seven numbers' (64), while the other is according to their biofunctions, such as ciRS-7 $(13,16)$, or their host genes, such as circ-ITCH $(77,92)$ and circMTO1 (120). Despite this, both methods have their own drawbacks. The former has a complex form that is not easily understood. The latter may provide the information of the gene that generates multiple circRNAs after transcription, and thus, this method is not used to name each circRNA based on its own characteristics.

CircRNAs were initially cognitively untranslatable, but recent reports have argued that some circRNAs carry ORFs, and that some of them contain IRES elements. As more evidence emerges, there is a growing interest in these functions and roles of circRNAs. It is predicted that this direction and trend in research regarding ORFs will become the new key area of interest in the field of circRNAs.

A recent study reported, for the first time, the presence of extensive circRNAs in exosomes (121), which are small membranous vesicles involved in endocytosis that are secreted by most types of cells (122). The sorting and loading of circRNAs into exosomes could be controlled by altering the associated miRNA levels in cells; these circRNAs may transfer to other recipient cells through biological activity. There is no doubt that circRNAs have potential as tumor biomarkers of clinical diagnostic and prognostic value. The above conclusions lay the foundations for a novel type of exosome-based cancer biomarker of the development of circRNAs and reveal the potential biological function of exosomal circRNAs. Research into circRNAs broadens our understanding of the eukaryotic genome and provides a new direction to investigate the comprehensive understanding of common diseases. Further studies on circRNAs may reveal biocontrol-inducing factors, the functions of different types of these molecules, whether circRNAs can interact directly with the genome (as with lncRNAs), and the kinetic mechanisms of circRNA degradation.

With the development of more advanced scientific technology and more in-depth study of circRNAs, future research may elucidate the physiological and pathological processes that circRNAs are involved in in the human body, the role of circRNAs in the blood as biomarkers and may further evaluate the biological functions of more unknown circRNAs. Authors report no competing interest in this work.

\section{Acknowledgements}

The authors thank Mr. Jia Liu for his suggestions on the structure and language editing of the whole article.

\section{Funding}

The Department of Thoracic Surgery, Affiliated Hospital of Qingdao University supported this study.

\section{Availability of data and materials}

Not applicable.

\section{Authors' contributions}

YW, TL, JL and WJ conceived and designed the study. YW wrote the paper. YW and QW jointly designed the figures. YW, TL, QW, JL and WJ reviewed and edited the manuscript. All authors read and approved the manuscript and agree to be accountable for all aspects of the research in ensuring that the accuracy or integrity of any part of the work are appropriately investigated and resolved.

\section{Ethics approval and consent to participate}

Not applicable.

\section{Patient consent for publication}

Not applicable.

\section{Competing interests}

No authors report any competing interest.

\section{References}

1. Amodio N, Raimondi L, Juli G, Stamato MA, Caracciolo D, Tagliaferri P and Tassone P: MALAT1: A druggable long non-coding RNA for targeted anti-cancer approaches. J Hematol Oncol 11: 63, 2018.

2. Glasgow AMA, De Santi C and Greene CM: Non-coding RNA in cystic fibrosis. Biochem Soc Trans 46: 619-630, 2018.

3. Zong L, Sun Q, Zhang H, Chen Z, Deng Y, Li D and Zhang L: Increased expression of circRNA_102231 in lung cancer and its clinical significance. Biomed Pharmacother 102: 639-644, 2018.

4. Soslau G: Circular RNA (circRNA) was an important bridge in the switch from the RNA world to the DNA world. J Theor Biol 447: 32-40, 2018.

5. Jiang XM, Li ZL, Li JL, Xu Y, Leng KM, Cui YF and Sun DJ: A novel prognostic biomarker for cholangiocarcinoma: circRNA Cdrlas. Eur Rev Med Pharmacol Sci 22: 365-371, 2018.

6. Zhang HD, Jiang LH, Sun DW, Hou JC and Ji ZL: CircRNA: A novel type of biomarker for cancer. Breast Cancer 25: 1-7, 2018.

7. Jeck WR, Sorrentino JA, Wang K, Slevin MK, Burd CE, Liu J, Marzluff WF and Sharpless NE: Circular RNAs are abundant, conserved, and associated with ALU repeats. RNA 19: 141-157, 2013.

8. Gao Y, Zhang J and Zhao F: Circular RNA identification based on multiple seed matching. Brief Bioinform: Feb 28, 2017 (Epub ahead of print). doi: 10.1093/bib/bbx014.

9. Lei K, Bai H, Wei Z, Xie C, Wang J, Li J and Chen Q: The mechanism and function of circular RNAs in human diseases. Exp Cell Res 368: 147-158, 2018.

10. Li D, Chen Y, Mei H, Jiao W, Song H, Ye L, Fang E, Wang X, Yang F, Huang K, et al: Ets-1 promoter-associated noncoding RNA regulates the NONO/ERG/Ets-1 axis to drive gastric cancer progression. Oncogene 37: 4871-4886, 2018.

11. Zhao ZJ and Shen J: Circular RNA participates in the carcinogenesis and the malignant behavior of cancer. RNA Biol 14: 514-521, 2017.

12. Hansen TB, Jensen TI, Clausen BH, Bramsen JB, Finsen B, Damgaard CK and Kjems J: Natural RNA circles function as efficient microRNA sponges. Nature 495: 384-388, 2013.

13. Memczak S, Jens M, Elefsinioti A, Torti F, Krueger J, Rybak A, Maier L, Mackowiak SD, Gregersen LH, Munschauer M, et al: Circular RNAs are a large class of animal RNAs with regulatory potency. Nature 495: 333-338, 2013.

14. Kulcheski FR, Christoff AP and Margis R: Circular RNAs are miRNA sponges and can be used as a new class of biomarker. J Biotechnol 238: 42-51, 2016.

15. Militello G, Weirick T, John D, Döring C, Dimmeler S and Uchida S: Screening and validation of lncRNAs and circRNAs as miRNA sponges. Brief Bioinform 18: 780-788, 2017. 
16. Salzman J, Chen RE, Olsen MN, Wang PL and Brown PO: Cell-type specific features of circular RNA expression. PLoS Genet 9: e1003777, 2013.

17. Kim J, Abdelmohsen K, Yang X, De S, Grammatikakis I, Noh JH and Gorospe M: LncRNA OIP5-AS1/cyrano sponges RNA-binding protein HuR. Nucleic Acids Res 44: 2378-2392, 2016.

18. Dai X, Zhang N, Cheng Y, Yang T, Chen Y, Liu Z, Wang Z, Yang $\mathrm{C}$ and Jiang Y: RNA-binding protein trinucleotide repeat-containing $6 \mathrm{~A}$ regulates the formation of circular RNA 0006916, with important functions in lung cancer cells. Carcinogenesis: May 3, 2018 (Epub ahead of print). doi: 10.1093/carcin/ bgy061.

19. Conn SJ, Pillman KA, Toubia J, Conn VM, Salmanidis M Phillips CA, Roslan S, Schreiber AW, Gregory PA and Goodall GJ: The RNA binding protein quaking regulates formation of circRNAs. Cell 160: 1125-1134, 2015.

20. Wu J, Jiang Z, Chen C, Hu Q, Fu Z, Chen J, Wang Z, Wang Q, Li A, Marks JR, et al: CircIRAK3 sponges miR-3607 to facilitate breast cancer metastasis. Cancer Lett 430: 179-192, 2018.

21. Yuan Y, Liu W, Zhang Y, Zhang Y and Sun S: CircRNA circ_0026344 as a prognostic biomarker suppresses colorectal cancer progression via microRNA-21 and microRNA-31. Biochem Biophys Res Commun 503: 870-875, 2018.

22. Zhou J, Wang H, Chu J, Huang Q, Li G, Yan Y, Xu T, Chen J and Wang Y: Circular RNA hsa_circ_0008344 regulates glioblastoma cell proliferation, migration, invasion, and apoptosis. J Clin Lab Anal: e22454, 2018.

23. Zhou LH, Yang YC, Zhang RY, Wang P, Pang MH and Liang LQ CircRNA_0023642 promotes migration and invasion of gastric cancer cells by regulating EMT. Eur Rev Med Pharmacol Sci 22 2297-2303, 2018

24. Hou LD and Zhang J: Circular RNAs: An emerging type of RNA in cancer. Int J Immunopathol Pharmacol 30: 1-6, 2017.

25. Jakobi T and Dieterich C: Deep computational circular RNA analytics from RNA-seq data. Methods Mol Biol 1724: 9-25, 2018.

26. Chen BJ, Byrne FL, Takenaka K, Modesitt SC, Olzomer EM, Mills JD, Farrell R, Hoehn KL and Janitz M: Analysis of the circular RNA transcriptome in endometrial cancer. Oncotarget 9: 5786-5796, 2018

27. Zhang Y, Zhang XO, Chen T, Xiang JF, Yin QF, Xing YH, Zhu S, Yang $\mathrm{L}$ and Chen LL: Circular intronic long noncoding RNAs. Mol Cell 51: 792-806, 2013.

28. Guo JU, Agarwal V, Guo H and Bartel DP: Expanded identification and characterization of mammalian circular RNAs. Genome Biol 15: 409, 2014

29. Salzman J, Gawad C, Wang PL, Lacayo N and Brown PO: Circular RNAs are the predominant transcript isoform from hundreds of human genes in diverse cell types. PLoS One 7: e30733, 2012.

30. Fumagalli MR, Zapperi S and La Porta CAM: Impact of the cross-talk between circular and messenger RNAs on cell regulation. J Theor Biol 454: 386-395, 2018.

31. Siede D, Rapti K, Gorska AA, Katus HA, Altmüller J, Boeckel JN, Meder B, Maack C, Völkers M, Müller OJ, et al: Identification of circular RNAs with host gene-independent expression in human model systems for cardiac differentiation and disease. J Mol Cell Cardiol 109: 48-56, 2017.

32. Wang Y and Wang Z: Efficient backsplicing produces translatable circular mRNAs. RNA 21: 172-179, 2015.

33. Ashwal-Fluss R, Meyer M, Pamudurti NR, Ivanov A, Bartok O, Hanan M, Evantal N, Memczak S, Rajewsky N, Kadener S, et al: circRNA biogenesis competes with pre-mRNA splicing. Mol Cell 56: 55-66, 2014.

34. Wilusz JE: Circular RNAs: Unexpected outputs of many protein-coding genes. RNA Biol 14: 1007-1017, 2017.

35. Hsiao KY, Sun HS and Tsai SJ: Circular RNA-new member of noncoding RNA with novel functions. Exp Biol Med 242: 1136-1141, 2017

36. Holdt LM, Kohlmaier A and Teupser D: Molecular roles and function of circular RNAs in eukaryotic cells. Cell Mol Life Sci 75: 1071-1098, 2018.

37. Petkovic S and Müller S: Synthesis and engineering of circular RNAs. Methods Mol Biol 1724: 167-180, 2018.

38. Li Z, Huang C, Bao C, Chen L, Lin M, Wang X, Zhong G, Yu B, $\mathrm{Hu}$ W, Dai L, et al: Exon-intron circular RNAs regulate transcription in the nucleus. Nat Struct Mol Biol 22: 256-264, 2015.

39. Chen L, Huang C, Wang X and Shan G: Circular RNAs in eukaryotic cells. Curr Genomics 16: 312-318, 2015.
40. Yang $\mathrm{Y}$ and Wang Z: Constructing GFP-based reporter to study back splicing and translation of circular RNA. Methods Mol Biol 1724: 107-118, 2018

41. Zhang XO, Dong R, Zhang Y, Zhang JL, Luo Z, Zhang J, Chen LL and Yang L: Diverse alternative back-splicing and alternative splicing landscape of circular RNAs. Genome Res 26: 1277-1287, 2016

42. Wang PL, Bao Y, Yee MC, Barrett SP, Hogan GJ, Olsen MN, Dinneny JR, Brown PO and Salzman J: Circular RNA is expressed across the eukaryotic tree of life. PLoS One 9: e90859, 2014.

43. Qu S, Yang X, Li X, Wang J, Gao Y, Shang R, Sun W, Dou K and Li H: Circular RNA: A new star of noncoding RNAs. Cancer Lett 365: 141-148, 2015 .

44. Rybak-Wolf A, Stottmeister C, Glažar P, Jens M, Pino N, Giusti S, Hanan M, Behm M, Bartok O, Ashwal-Fluss R, et al: Circular RNAs in the mammalian brain are highly abundant, conserved, and dynamically expressed. Mol Cell 58: 870-885, 2015.

45. Schmitz KM, Mayer C, Postepska A and Grummt I: Interaction of noncoding RNA with the rDNA promoter mediates recruitment of DNMT3b and silencing of rRNA genes. Genes Dev 24: 2264-2269, 2010

46. Beckedorff FC, Ayupe AC, Crocci-Souza R, Amaral MS, Nakaya HI, Soltys DT, Menck CF, Reis EM and VerjovskiAlmeida S: The intronic long noncoding RNA ANRASSF1 recruits PRC2 to the RASSF1A promoter, reducing the expression of RASSF1A and increasing cell proliferation. PLoS Genet 9: e1003705, 2013.

47. Müller S and Appel B: In vitro circularization of RNA. RNA Biol 14: 1018-1027, 2017.

48. Zhao F, Han Y, Liu Z, Zhao Z, Li Z and Jia K: circFADS2 regulates lung cancer cells proliferation and invasion via acting as a sponge of miR-498. Biosci Rep 38: pii: BSR20180570, 2018.

49. Lin X and Chen Y: Identification of potentially functional CircRNA-miRNA-mRNA regulatory network in hepatocellular carcinoma by integrated microarray analysis. Med Sci Monit Basic Res 24: 70-78, 2018.

50. Zhang M, Jia L and Zheng Y: circRNA expression profiles in human bone marrow stem cells undergoing osteoblast differentiation. Stem Cell Rev: Jul 25, 2018 (Epub ahead of print). doi: 10.1007/s12015-018-9841-x.

51. Li S, Sun X, Miao S, Lu T, Wang Y, Liu J and Jiao W: hsa_circ_0000729, a potential prognostic biomarker in lung adenocarcinoma. Thorac Cancer 9: 924-930, 2018

52. Haque S and Harries LW: Circular RNAs (circRNAs) in health and disease. Genes 8: E353, 2017.

53. Schneider T, Hung LH, Schreiner S, Starke S, Eckhof H, Rossbach O, Reich S, Medenbach J and Bindereif A: CircRNA-protein complexes: IMP3 protein component defines subfamily of circRNPs. Sci Rep 6: 31313, 2016.

54. Ghosal S, Das S, Sen R, Basak P and Chakrabarti J: Circ2Traits: A comprehensive database for circular RNA potentially associated with disease and traits. Front Genet 4: 283, 2013.

55. Gehring NH, Kunz JB, Neu-Yilik G, Breit S, Viegas MH, Hentze MW and Kulozik AE: Exon-junction complex components specify distinct routes of nonsense-mediated mRNA decay with differential cofactor requirements. Mol Cell 20: 65-75, 2005.

56. Dudekula DB, Panda AC, Grammatikakis I, De S, Abdelmohsen K and Gorospe M: CircInteractome: A web tool for exploring circular RNAs and their interacting proteins and microRNAs. RNA Biol 13: 34-42, 2016.

57. Perriman R and Ares M Jr: Circular mRNA can direct translation of extremely long repeating-sequence proteins in vivo. RNA 4: 1047-1054, 1998

58. AbouHaidar MG, Venkataraman S, Golshani A, Liu B and Ahmad T: Novel coding, translation, and gene expression of a replicating covalently closed circular RNA of $220 \mathrm{nt}$. Proc Natl Acad Sci USA 111: 14542-14547, 2014.

59. Chen CY and Sarnow P: Initiation of protein synthesis by the eukaryotic translational apparatus on circular RNAs. Science 268: 415-417, 1995.

60. Chen X, Han P, Zhou T, Guo X, Song X and Li Y: circRNADb: A comprehensive database for human circular RNAs with protein-coding annotations. Sci Rep 6: 34985, 2016.

61. Jeck WR and Sharpless NE: Detecting and characterizing circular RNAs. Nat Biotechnol 32: 453-461, 2014.

62. Yang Y, Fan X, Mao M, Song X, Wu P, Zhang Y, Jin Y, Yang Y, Chen LL, Wang Y, et al: Extensive translation of circular RNAs driven by $\mathrm{N}^{6}$-methyladenosine. Cell Res 27: 626-641, 2017. 
63. Lasda E and Parker R: Circular RNAs co-precipitate with extracellular vesicles: A possible mechanism for circRNA clearance. PLoS One 11: e0148407, 2016.

64. Glažar P, Papavasileiou P and Rajewsky N: circBase: A database for circular RNAs. RNA 20: 1666-1670, 2014.

65. Liu YC, Li JR, Sun CH, Andrews E, Chao RF, Lin FM, Weng SL, Hsu SD, Huang CC, Cheng C, et al: CircNet: A database of circular RNAs derived from transcriptome sequencing data. Nucleic Acids Res 44: D209-D215, 2016.

66. Burd CE, Jeck WR, Liu Y, Sanoff HK, Wang Z and Sharpless NE: Expression of linear and novel circular forms of an INK4/ARF-associated non-coding RNA correlates with atherosclerosis risk. PLoS Genet 6: e1001233, 2010.

67. He J, Xie Q, Xu H, Li J and Li Y: Circular RNAs and cancer. Cancer Lett 396: 138-144, 2017.

68. Wang F, Nazarali AJ and Ji S: Circular RNAs as potential biomarkers for cancer diagnosis and therapy. Am J Cancer Res 6: 1167-1176, 2016.

69. Kent OA and Mendell JT: A small piece in the cancer puzzle: microRNAs as tumor suppressors and oncogenes. Oncogene 25: 6188-6196, 2006.

70. Peng Y and Croce CM: The role of MicroRNAs in human cancer. Signal Transduct Target Ther 1: 15004, 2016.

71. Zheng Q, Bao C, Guo W, Li S, Chen J, Chen B, Luo Y, Lyu D, Li Y, Shi G, et al: Circular RNA profiling reveals an abundant circHIPK3 that regulates cell growth by sponging multiple miRNAs. Nat Commun 7: 11215, 2016.

72. Scotti MM and Swanson MS: RNA mis-splicing in disease. Nat Rev Genet 17: 19-32, 2016.

73. Li P, Chen S, Chen H, Mo X, Li T, Shao Y, Xiao B and Guo J: Using circular RNA as a novel type of biomarker in the screening of gastric cancer. Clin Chim Acta 444: 132-136, 2015

74. Chen J, Li Y, Zheng Q, Bao C, He J, Chen B, Lyu D, Zheng B, $\mathrm{Xu}$ Y, Long Z, et al: Circular RNA profile identifies circPVT1 as a proliferative factor and prognostic marker in gastric cancer. Cancer Lett 388: 208-219, 2017.

75. Li P, Chen H, Chen S, Mo X, Li T, Xiao B, Yu R and Guo J: Circular RNA 0000096 affects cell growth and migration in gastric cancer. Br J Cancer 116: 626-633, 2017.

76. Huang G, Zhu H, Shi Y, Wu W, Cai H and Chen X: cir-ITCH plays an inhibitory role in colorectal cancer by regulating the Wnt/ß-catenin pathway. PLoS One 10: e0131225, 2015.

77. Wan L, Zhang L, Fan K, Cheng ZX, Sun QC and Wang JJ: Circular RNA-ITCH suppresses lung cancer proliferation via inhibiting the Wnt $/ \beta$-catenin pathway. Biomed Res Int 2016 : 1579490, 2016.

78. Yao JT, Zhao SH, Liu QP, Lv MQ, Zhou DX, Liao ZJ and Nan KJ Over-expression of CircRNA_100876 in non-small cell lung cancer and its prognostic value. Pathol Res Pract 213: 453-456, 2017.

79. Bachmayr-Heyda A, Reiner AT, Auer K, Sukhbaatar N, Aust S, Bachleitner-Hofmann T, Mesteri I, Grunt TW, Zeillinger R and Pils D: Correlation of circular RNA abundance with proliferation - exemplified with colorectal and ovarian cancer, idiopathic lung fibrosis, and normal human tissues. Sci Rep 5: 8057, 2015.

80. Xie H, Ren X, Xin S, Lan X, Lu G, Lin Y, Yang S, Zeng Z, Liao W, Ding YQ, et al: Emerging roles of circRNA_001569 targeting miR-145 in the proliferation and invasion of colorectal cancer. Oncotarget 7: 26680-26691, 2016.

81. Li JH, Liu S, Zhou H, Qu LH and Yang JH: starBase v2.0: Decoding miRNA-ceRNA, miRNA-ncRNA and protein-RNA interaction networks from large-scale CLIP-Seq data. Nucleic Acids Res 42: D92-D97, 2014.

82. Jiang Y, Yim SH, Xu HD, Jung SH, Yang SY, Hu HJ, Jung CK and Chung YJ: A potential oncogenic role of the commonly observed E2F5 overexpression in hepatocellular carcinoma. World J Gastroenterol 17: 470-477, 2011.

83. Annunziata CM, Kleinberg L, Davidson B, Berner A, Gius D, Tchabo N, Steinberg SM and Kohn EC: BAG-4/SODD and associated antiapoptotic proteins are linked to aggressiveness of epithelial ovarian cancer. Clin Cancer Res 13: 6585-6592, 2007.

84. Ozawa F, Friess H, Zimmermann A, Kleeff J and Büchler MW: Enhanced expression of Silencer of death domains (SODD/BAG-4) in pancreatic cancer. Biochem Biophys Res Commun 271: 409-413, 2000.

85. Li Y, Zhu X, Zeng Y, Wang J, Zhang X, Ding YQ and Liang L: FMNL2 enhances invasion of colorectal carcinoma by inducing epithelial-mesenchymal transition. Mol Cancer Res 8: 1579-1590, 2010.
86. Liang L, Li X, Zhang X, Lv Z, He G, Zhao W, Ren X, Li Y, Bian X, Liao W, et al: MicroRNA-137, an HMGA1 target, suppresses colorectal cancer cell invasion and metastasis in mice by directly targeting FMNL2. Gastroenterology 144: 624-635.e4, 2013.

87. Zhu M, Xu Y, Chen Y and Yan F: Circular BANP, an upregulated circular RNA that modulates cell proliferation in colorectal cancer. Biomed Pharmacother 88: 138-144, 2017.

88. Guo JN, Li J, Zhu CL, Feng WT, Shao JX, Wan L, Huang MD and He JD: Comprehensive profile of differentially expressed circular RNAs reveals that hsa_circ_0000069 is upregulated and promotes cell proliferation, migration, and invasion in colorectal cancer. Onco Targets Ther 9: 7451-7458, 2016.

89. Wang X, Zhang Y, Huang L, Zhang J, Pan F, Li B, Yan Y, Jia B, Liu H, Li S and Zheng W: Decreased expression of hsa circ 001988 in colorectal cancer and its clinical significances. Int J Clin Exp Pathol 8: 16020-16025, 2015.

90. Cortés-López M and Miura P: Emerging functions of circular RNAs. Yale J Biol Med 89: 527-537, 2016.

91. Hsiao KY, Lin YC, Gupta SK, Chang N, Yen L, Sun HS and Tsai SJ: Noncoding effects of circular RNA CCDC66 promote colon cancer growth and metastasis. Cancer Res 77: 2339-2350, 2017.

92.Li F, Zhang L, Li W, Deng J, Zheng J, An M, Lu J and Zhou Y: Circular RNA ITCH has inhibitory effect on ESCC by suppressing the $\mathrm{Wnt} / \beta$-catenin pathway. Oncotarget 6 : 6001-6013, 2015.

93. Su H, Lin F, Deng X, Shen L, Fang Y, Fei Z, Zhao L, Zhang X, Pan H, Xie D, et al: Profiling and bioinformatics analyses reveal differential circular RNA expression in radioresistant esophageal cancer cells. J Transl Med 14: 225, 2016.

94. Xia W, Qiu M, Chen R, Wang S, Leng X, Wang J, Xu Y, Hu J, Dong G, Xu PL and Yin R: Circular RNA has_circ_0067934 is upregulated in esophageal squamous cell carcinoma and promoted proliferation. Sci Rep 6: 35576, 2016.

95. Pinder SE: Ductal carcinoma in situ (DCIS): Pathological features, differential diagnosis, prognostic factors and specimen evaluation. Mod Pathol 23 (Suppl 2): S8-S13, 2010.

96. Hernandez L, Wilkerson PM, Lambros MB, Campion-Flora A, Rodrigues DN, Gauthier A, Cabral C, Pawar V, Mackay A, $\mathrm{A}^{\prime}$ Hern R, et al: Genomic and mutational profiling of ductal carcinomas in situ and matched adjacent invasive breast cancers reveals intra-tumour genetic heterogeneity and clonal selection. J Pathol 227: 42-52, 2012.

97. Galasso M, Costantino G, Pasquali L, Minotti L, Baldassari F, Corrà $\mathrm{F}$, Agnoletto $\mathrm{C}$ and Volinia $\mathrm{S}$ : Profiling of the predicted circular RNAs in ductal in situ and invasive breast cancer: A pilot study. Int J Genomics 2016: 4503840, 2016.

98. Gregory PA, Bert AG, Paterson EL, Barry SC, Tsykin A, Farshid G, Vadas MA, Khew-Goodall Y and Goodall GJ: The miR-200 family and miR-205 regulate epithelial to mesenchymal transition by targeting ZEB1 and SIP1. Nat Cell Biol 10: 593-601, 2008.

99. Ye ZB, Ma G, Zhao YH, Xiao Y, Zhan Y, Jing C, Gao K, Liu ZH and Yu SJ: miR-429 inhibits migration and invasion of breast cancer cells in vitro. Int J Oncol 46: 531-538, 2015.

100. Yang W, Du WW, Li X, Yee AJ and Yang BB: Foxo3 activity promoted by non-coding effects of circular RNA and Foxo3 pseudogene in the inhibition of tumor growth and angiogenesis. Oncogene 35: 3919-3931, 2016.

101. Du WW, Ling F, Yang W, Wu N, Awan FM, Yang Z and Yang BB: Induction of tumor apoptosis through a circular RNA enhancing Foxo3 activity. Cell Death Differ 24: 357-370, 2017.

102. Caiment F, Gaj S, Claessen S and Kleinjans J: High-throughput data integration of RNA-miRNA-circRNA reveals novel insights into mechanisms of benzo[a]pyrene-induced carcinogenicity. Nucleic Acids Res 43: 2525-2534, 2015.

103. Shang X, Li G, Liu H, Li T, Liu J, Zhao Q and Wang C: Comprehensive circular RNA profiling reveals that hsa circ 0005075, a new circular rna biomarker, is involved in hepatocellular crcinoma development. Medicine 95: e3811, 2016.

104. Dong L, Deng J, Sun ZM, Pan AP, Xiang XJ, Zhang L, Yu F, Chen J, Sun Z, Feng M and Xiong JP: Interference with the $\beta$-catenin gene in gastric cancer induces changes to the miRNA expression profile. Tumour Biol 36: 6973-6983, 2015.

105. Warnecke-Eberz U, Chon SH, Hölscher AH, Drebber U and Bollschweiler E: Exosomal onco-miRs from serum of patients with adenocarcinoma of the esophagus: Comparison of miRNA profiles of exosomes and matching tumor. Tumour Biol 36: 4643-4653, 2015 
106. Qin M, Liu G, Huo X, Tao X, Sun X, Ge Z, Yang J, Fan J, Liu L and Qin W: Hsa_circ_0001649: A circular RNA and potential novel biomarker for hepatocellular carcinoma. Cancer Biomark 16: 161-169, 2016.

107. Yu L, Gong X, Sun L, Zhou Q, Lu B and Zhu L: The circular RNA Cdrlas act as an oncogene in hepatocellular carcinoma through targeting miR-7 expression. PLoS One 11: e0158347, 2016.

108. Xu L, Zhang M, Zheng X, Yi P, Lan C and Xu M: The circular RNA ciRS-7 (Cdrlas) acts as a risk factor of hepatic microvascular invasion in hepatocellular carcinoma. J Cancer Res Clin Oncol 143: 17-27, 2017.

109. Siegel RL, Miller KD and Jemal A: Cancer statistics, 2016. CA Cancer J Clin 66: 7-30, 2016.

110. Zhong Z, Lv M and Chen J: Screening differential circular RNA expression profiles reveals the regulatory role of circTCF25-miR-103a-3p/miR-107-CDK6 pathway in bladder carcinoma. Sci Rep 6: 30919, 2016.

111. Guarnerio J, Bezzi M, Jeong JC, Paffenholz SV, Berry K, Naldini MM, Lo-Coco F, Tay Y, Beck AH and Pandolfi PP: Oncogenic role of fusion-circrnas derived from cancer-associated chromosomal translocations. Cell 165: 289-302, 2016.

112. Alhasan AA, Izuogu OG, Al-Balool HH, Steyn JS, Evans A, Colzani M, Ghevaert C, Mountford JC, Marenah L, Elliott DJ, et al: Circular RNA enrichment in platelets is a signature of transcriptome degradation. Blood 127: e1-e11, 2016.

113. Best MG, Sol N, Kooi I, Tannous J, Westerman BA, Rustenburg F, Schellen P, Verschueren H, Post E, Koster J, et al: RNA-Seq of tumor-educated platelets enables blood-based pan-cancer, multiclass, and molecular pathway cancer diagnostics. Cancer Cell 28: 666-676, 2015.

114. Liu X, Zhong Y, Li J and Shan A: Circular RNA circ-NT5C2 acts as an oncogene in osteosarcoma proliferation and metastasis through targeting miR-448. Oncotarget 8: 114829-114838, 2017.
115. Kun-Peng Z, Xiao-Long M and Chun-Lin Z: Overexpressed circPVT1, a potential new circular RNA biomarker, contributes to doxorubicin and cisplatin resistance of osteosarcoma cells by regulating ABCB1. Int J Biol Sci 14: 321-330, 2018.

116. Qu S, Song W, Yang X, Wang J, Zhang R, Zhang Z, Zhang H and Li H: Microarray expression profile of circular RNAs in human pancreatic ductal adenocarcinoma. Genom Data 5: 385-387, 2015.

117. Sand M, Bechara FG, Gambichler T, Sand D, Bromba M, Hahn SA, Stockfleth E and Hessam S: Circular RNA expression in cutaneous squamous cell carcinoma. J Dermatol Sci 83: 210-218, 2016.

118. Peng N, Shi L, Zhang Q, Hu Y, Wang N and Ye H: Microarray profiling of circular RNAs in human papillary thyroid carcinoma. PLoS One 12: e0170287, 2017.

119. Xuan L, Qu L, Zhou H, Wang P, Yu H, Wu T, Wang X, Li Q Tian L, Liu M and Sun Y: Circular RNA: A novel biomarker for progressive laryngeal cancer. Am J Transl Res 8: 932-939, 2016.

120. Han D, Li J, Wang H, Su X, Hou J, Gu Y, Qian C, Lin Y, Liu X, Huang M, et al: Circular RNA circMTO1 acts as the sponge of microRNA-9 to suppress hepatocellular carcinoma progression. Hepatology 66: 1151-1164, 2017.

121. Li Y, Zheng Q, Bao C, Li S, Guo W, Zhao J, Chen D, Gu J, He X and Huang S: Circular RNA is enriched and stable in exosomes: A promising biomarker for cancer diagnosis. Cell Res 25: 981-984,2015.

122. Melo SA, Luecke LB, Kahlert C, Fernandez AF, Gammon ST, Kaye J, LeBleu VS, Mittendorf EA, Weitz J, Rahbari N, et al: Glypican-1 identifies cancer exosomes and detects early pancreatic cancer. Nature 523: 177-182, 2015.

This work is licensed under a Creative Commons Attribution-NonCommercial-NoDerivatives 4.0 International (CC BY-NC-ND 4.0) License. 